Published in final edited form as:

Nat Genet. 2016 October ; 48(10): 1171-1184. doi:10.1038/ng.3667.

\title{
The genetics of blood pressure regulation and its target organs from association studies in 342,415 individuals
}

\author{
A full list of authors and affiliations appears at the end of the article. \\ \# These authors contributed equally to this work.
}

\begin{abstract}
To dissect the genetic architecture of blood pressure and assess effects on target-organ damage, we analyzed 128,272 SNPs from targeted and genome-wide arrays in 201,529 individuals of European ancestry and genotypes from an additional 140,886 individuals were used for validation. We identified 66 blood pressure loci, of which 17 were novel and 15 harbored multiple distinct association signals. The 66 index SNPs were enriched for cis-regulatory elements, particularly in vascular endothelial cells, consistent with a primary role in blood pressure control through modulation of vascular tone across multiple tissues. The 66 index SNPs combined in a risk score showed comparable effects in 64,421 individuals of non-European descent. The 66-SNP blood pressure risk score was significantly associated with target-organ damage in multiple tissues, with minor effects in the kidney. Our findings expand current knowledge of blood pressure pathways and highlight tissues beyond the classic renal system in blood pressure regulation.
\end{abstract}

\section{INTRODUCTION}

There are considerable physiological, clinical and genetic data that point to the kidney as the major regulator of blood pressure (BP) and to renal damage as a consequence of long-term BP elevation. However, alternative hypotheses, such as increasing systemic vascular resistance, are also serious contenders to explain the rise of BP with increasing age, but with limited genetic support. The genetic basis of elevated blood pressure or hypertension (HTN) involves many loci that have been identified using large-scale analyses of candidate genes ${ }^{1,2}$, linkage studies, and genome-wide association studies (GWAS) ${ }^{3-12}$. The genes underlying BP regulation can help resolve many of the open questions regarding BP (patho-) physiology.

\footnotetext{
Users may view, print, copy, and download text and data-mine the content in such documents, for the purposes of academic research, subject always to the full Conditions of use:http://www.nature.com/authors/editorial_policies/license.html\#terms

Corresponding authors: Christopher Newton-Cheh (cnewtoncheh@mgh.harvard.edu) and Patricia B. Munroe (p.b.munroe@qmul.ac.uk).

\#These authors jointly supervised this work.

${ }^{57}$ A list of members and affiliations appears in the Supplementary Note

SUPPLEMENTARY NOTE

Supplementary Note is available in the online version of the paper.

URLs

http://hgdownload.cse.ucsc.edu/goldenPath/hg19/encodeDCC/wgEncodeUwDnase for enrichment analyses. Accessed 3/13/2013. http://www.genome.gov/gwastudies for enrichment analyses. Accessed 3/13/2013.

http://genome.ucsc.edu/ENCODE/cellTypes.html for enrichment analyses. Accessed 3/13/2013.

COMPETING FINANCIAL INTERESTS

The authors declare competing financial interests (see corresponding section in the Supplementary Note).
} 
While $\sim 40-50 \%$ of BP variability is heritable ${ }^{13,14}$, the genetic variation identified to date explains only $\sim 2 \%{ }^{1-12}$.

The Cardio-MetaboChip is a custom genotyping microarray designed to facilitate costeffective follow-up of nominal associations for metabolic and cardiovascular traits, including BP. This array comprises 196,725 variants, including 5,000 SNPs with nominal $(P<0.016)$ evidence of BP association in our previous GWAS meta-analysis ${ }^{5}$. Furthermore, the array includes several dense scaffolds for fine mapping of selected loci spanning, on average, genomic regions of 350 kilobases $^{5,16}$, of which 24 include genome-wide significant BP association in the current study ${ }^{5,16}$.

\section{RESULTS}

\section{Novel genetic loci associated with systolic and diastolic BP}

We performed meta-analyses of association summary statistics from a total of 201,529 individuals of European (EUR) ancestry from 74 studies: (i) 109,096 individuals from 46 studies genotyped on Cardio-MetaboChip; and (ii) 92,433 individuals from 28 studies with imputed genotype data from genome-wide genotyping at variants included on the CardioMetaboChip. Twenty-four of the 28 studies with genome-wide genotyping data had contributed to previous analyses (Supplementary Tables 1-3) ${ }^{5,7}$.

BP was measured using standardized protocols in all studies ${ }^{5,17}$ (Supplementary Table 1, Online methods). Association statistics for systolic and diastolic BP (SBP and DBP) in models adjusting for age, age ${ }^{2}$, sex, and body mass index (BMI), were obtained for each study separately, with study-specific genomic control applied to correct for possible population structure. Fixed-effects meta-analysis proceeded in 4 stages, separately for the following SNP associations: Stage 1, using results based on 46 studies using CardioMetaboChip genotypes of 109,096 participants; Stage 2, using additional results based on imputed genotypes from genome-wide genotyping arrays in 4 previously unpublished studies; Stage 3 using imputed genotypes from genome-wide genotyping arrays in 24 previously published studies ${ }^{5}$; and Stage 4, the joint meta-analysis of Stages 1-3 including a total of 201,529 independent individuals (Supplementary Figure 1, Supplementary Tables 2-3, Supplementary Note). To account for population structure between studies in Stages 1-3 of our meta-analysis, genomic control correction was applied to meta-analysis results from each of these stages in an approach aggregating summary statistics from GWAS and CardioMetaboChip studies ${ }^{18,19}$.

After stage 4, 67 loci attained genome-wide significance $\left(P<5 \times 10^{-8}\right), 18$ of which were not previously reported in the literature (Supplementary Table 4). Quantile-quantile plots of the stage 4 meta-analysis showed an excess of small $P$ values, with an elevated genomic control lambda estimate that was persistent, albeit attenuated, after excluding all 66 loci

(Supplementary Figure 2). This observation is compatible with either residual uncorrected population stratification or the presence of a large number of variants that are truly associated with BP but fail to achieve genome-wide significance in the current metaanalysis. The Cardio-MetaboChip array's inclusion of SNPs from a prior BP GWAS ${ }^{5}$ does not appear to be the sole explanation, as we did not observe a significant decrease of the 
excess of small $P$ values after exclusion of all SNPs that were included on the CardioMetaboChip based on nominal BP association (Supplementary Figures 3 and 4). Since the quantile-quantile plots continued to show deviation from the null expectation, we sought additional validation for 18 variants attaining genome-wide significance, but without prior support in the literature, in up to 140,886 individuals of European ancestry from UK Biobank $^{20}$. For these SNPs, we performed a stage 5 meta-analysis combining the association summary statistics from stage 4 and UK Biobank, in a total of up to 342,415 individuals (Supplementary Table 5).

Upon stage 5 meta-analysis, 17 of 18 variants retained genome-wide significance for the primary trait (SBP or DBP result with the lower $P$ value). The one variant that was not genome-wide significant had a borderline $P$ value of $4.49 \times 10^{-8}$ at stage 4 . These findings are consistent with appropriate calibration of the association test statistics at stage 4 such that observing one failure among 18 validation tests is consistent with the use of a threshold $\left(P<5 \times 10^{-8}\right)$ designed to have a 1 in 20 chance of a result as or more extreme solely due to chance. In total, 66 loci attained genome-wide significance: 13 loci for SBP only, 12 loci for DBP only, and 41 loci for both traits. Of these, 17 BP loci were novel, while 49 were previously reported at genome-wide significance (Table 1 and Figure 1).

Compared with previously reported BP variants ${ }^{5,7,21}$, the average absolute effect size of the newly discovered variants is smaller, with comparable minor allele frequency (MAF), presumably owing to the increased power of a larger sample size (Table 2). As expected from the high correlation between SBP and DBP effects, the observed directions of effects for the two traits were generally concordant (Supplementary Figure 5), and the absolute effect sizes were inversely correlated with MAF (Table 1 and Supplementary Figure 6). The 66 BP SNPs explained 3.46\% and 3.36\% of SBP and DBP variance, respectively, a modest increase from $2.95 \%$ and $2.78 \%$ for SBP and DBP, respectively, for the 49 previously reported SNPs (Supplementary Note). The low percent variance explained is consistent with estimates that large numbers of common variants with weak effects at a large number of loci influence $\mathrm{BP}^{5}$.

\section{Signal refinement at the 66 BP loci}

To identify distinct signals of association at the $66 \mathrm{BP}$ loci and the variants most likely to be causal for each, we started with an approximate conditional analysis using a model selection procedure implemented in the GCTA-COJO package ${ }^{22,23}$ as well as a detailed literature review of all published BP association studies. GCTA-COJO analysis was performed using the association summary statistics for SBP and DBP from the Stage 4 EUR ancestry metaanalyses, with the linkage disequilibrium (LD) between variants estimated on the basis of Cardio-MetaboChip genotype data from 7,006 individuals of EUR ancestry from the GoDARTS cohort ${ }^{24}$. More than one distinct BP association signal was identified at 13 loci at $P<5 \times 10^{-8}$ (Supplementary Table 6, Supplementary Figures 7, and Supplementary Note). At six loci, the distinct signals were identified for both SBP and DBP analyzed separately; these trait-specific associations were represented by the same or highly correlated $\left(\mathrm{r}^{2}>0.8\right)$ SNPs at 5 of the 6 loci (Supplementary Tables 7 and 8). We repeated GCTA-COJO analyses using the same summary association results, but with a different 
reference sample for LD estimates (WTCCC1-T2D/58BC, N = 2,947, Supplementary

Note) and observed minimal differences arising from minor fluctuations in the association $P$ value in the joint regression models (Supplementary Tables 7 and 8). LD-based comparisons of published association signals at established BP loci, and the current study's findings suggested that at 10 loci, the signals identified by the single-SNP and the GCTACOJO analyses were distinct from those reported in the literature (Supplementary Table 9).

We then performed multivariable regression modeling in a single large cohort (Women's Genome Health Study, WGHS, N = 23,047) with simultaneous adjustment for both 1) all combinations of putative index SNPs for each distinct signal from the GCTA-COJO conditional analyses, and 2) all index SNPs for all potential distinct signals identified by our literature review (Supplementary Table 9, Supplementary Note). Although WGHS is very large as a single study, power is reduced in a single sample compared to that in the overall meta-analysis (23k vs. $342 \mathrm{k}$ individuals) and consequently the failure to reach significance does not represent non-replication for individual SNPs. The WGHS analysis supported two distinct association signals at eight of 13 loci identified in the GCTA-COJO analysis, but could not provide support for the remaining five (Supplementary Table 10). The joint SNP modeling in WGHS additionally supported two distinct signals of association at three other loci ( $G U C Y 1 A 3-G U C Y 1 B 3, S Y N P O 2 L$ and $T B X 5-T B X 3$ ), at which the SNP identified in the current study is distinct from that previously reported in the literature ${ }^{5,11}$.

We sought to refine the localization of likely functional variants at loci with high-density coverage on the Cardio-MetaboChip. We followed a Bayesian approach to define, for each signal, credible sets of variants that have $99 \%$ probability of containing or tagging the causal variant (Supplementary Note). To improve the resolution of the method, the analyses were restricted to 24 regions selected to fine map (FM) genetic associations, and that included at least one SNP reaching genome-wide significance in the current meta-analyses (Supplementary Table 11). Twenty-one of the Cardio-MetaboChip FM regions were BP loci in the original design, with three of the newly discovered BP loci in FM regions that were originally selected for other non-BP traits. We observed that the $99 \%$ credible SNP sets at five BP loci spanned $<20 \mathrm{~kb}$. The greatest refinement was observed at the $S L C 39 A 8$ locus for SBP and DBP, and at the $Z C 3 H C 1$ and PLCE1 loci for DBP, where the $99 \%$ credible sets included only the index variants (Supplementary Table 12). Although SNPs in credible sets were primarily non-coding, they included one synonymous and seven non-synonymous variants that attained high posterior probability of driving seven distinct association signals at six BP loci (Supplementary Table 12). Of these, three variants alone account for more than $95 \%$ of the posterior probability of driving the association signal observed at each of three loci (Supplementary Table 12 and 13). Despite reduced statistical power, the analyses restricted to the samples with Cardio-MetaboChip genotypes only $(\mathrm{N}=109,096)$ identified the majority of SNPs identified in the GWAS+Cardio-MetaboChip data (Supplementary Table 12). The full list of SNPs in the 99\% credible sets are listed in Supplementary Table 13. 


\section{What do the BP variants do?}

Index SNPs or their proxies $\left(r^{2}>0.8\right)$ altered amino acid sequence at 11 of 66 BP loci (Table 1). Thus, the majority of BP-association signals are likely driven by non-coding variants hypothesized to regulate expression of some nearby gene in cis. To characterize their effects, we first sought SNPs associated with gene expression (eSNPs) from a range of available expression data which included hypertension target end organs and cells of the circulatory system (heart tissue, kidney tissue, brain tissue, aortic endothelial cells, blood vessels) and other tissue/cell types ( $\left(\mathrm{CD}^{+}{ }^{+}\right.$macrophages, monocytes lymphoblastoid cell lines, skin tissue, fat tissue, and liver tissue). Fourteen BP-associated SNPs at the MTHFRNPPB, MDM4, ULK4, CYP1A1-ULK3, ADM, FURIN-FES, FIGN, and PSMD5 loci were eSNPs across different tissues (Supplementary Table 14). Of these 14 eSNPs, three were also predicted to alter the amino acid sequence at the MTHFR-NPPB, MAP4 and ULK4 loci, providing two potential mechanisms to explore in functional studies. Second, we used gene expression levels measured in whole blood in two different samples each including $>5,000$ individuals of EUR descent. We tested whether the lead BP SNP was associated with expression of any transcript in cis $(<1 \mathrm{Mb}$ from the lead SNP at each locus) at a false discovery rate (FDR) of $<0.05$, accounting for all possible cis-transcript association tests genome-wide. It is likely that we did not genotype the causal genetic variant underlying each BP association signal; a nearby SNP-transcript association, due to LD, may therefore reflect an independent genetic effect on expression that is unrelated to the BP effect. Consequently, we assumed that the lead BP SNP and the most significant eSNP for a given transcript should be highly correlated $\left(r^{2}>0.7\right)$. Furthermore, we assumed that the significance of the transcript association with the lead BP SNP should be substantially reduced in a conditional model adjusting for the best eSNP for a given transcript. Eighteen SNPs at 15 loci were associated with 22 different transcripts, with a total of 23 independent SNP-transcript associations (three SNPs were associated with two transcripts each, Supplementary Table 15, Supplementary Note). The genes expressed in a BP SNP allele-specific manner are clearly high-priority candidates to mediate the BP association. In whole blood, these genes included obvious biological candidates such as $G U C Y 1 A 3$, encoding the alpha subunit of the soluble guanylate cyclase protein, and $A D M$, encoding adrenomedullin, both of which are known to induce vasodilation ${ }^{25,26}$. There was some overlap of eSNPs between the whole blood and other tissue datasets at the MTHFR-NPPB, MDM4, PSMD5, ULK4 and CYP1A1-ULK3 loci, illustrating additional potentially causal genes for further study.

An alternative method for understanding the effect on BP of non-coding variants is to determine whether they fall within DNaseI hypersensitivity sites (DHSs). We performed two analyses to investigate whether BP SNPs or their LD proxies $\left(r^{2}>0.8\right)$ were enriched in DHSs in a cell-type-specific manner (Supplementary Note). First, we used Epigenomics Roadmap and ENCODE DHS data from 123 adult cell lines or tissues ${ }^{27-29}$ to estimate the fold increase in the proportion of BP SNPs mapping to DHSs compared to SNPs associated at genome-wide significance with non-BP phenotypes from the NHGRI GWAS catalog ${ }^{30}$. We observed that 7 out of the 10 cell types with the greatest relative enrichment of BP SNPs mapping to DHSs were from blood vessels (vascular or micro-vascular endothelial cell-lines or cells) and 11 of the 12 endothelial cells were among the top quarter most enriched among the 123 cell types (Figure 2 and Supplementary Table 16). In a second analysis of an 
expanded set of tissues and cell lines, in which cell types were grouped into tissues

(Supplementary Table 17), BP-associated SNP enrichment in DHSs in blood vessels was again observed $\left(P=1.2 \times 10^{-9}\right)$, as well as in heart samples $\left(P=5.3 \times 10^{-8}\right.$;

Supplementary Table 18).

We next tested whether there was enrichment of BP SNPs in H3K4me $3^{31}$ sites, a methylation mark associated with both promoter and enhancer DNA. We observed significant enrichment in a range of cell types including CD34 primary cells, adult kidney cells, and muscle satellite cultured cells(Supplementary Table 19). Enrichment of BP SNPs in predicted strong and weak enhancer states and in active promoters ${ }^{32}$ in a range of cell types was also observed (Supplementary Table 20, Supplementary Figure 8).

We used Meta-Analysis Gene-set Enrichment of variaNT Associations (MAGENTA) ${ }^{33}$ to attempt to identify pathways over-represented in the BP association results. No gene sets meeting experiment-wide significance for enrichment for BP association were identified by MAGENTA after correction for multiple testing, although some attained nominal significance (Supplementary Table 21, Supplementary Note). We also adapted the DEPICT $^{34}$ pathway analysis tool (Data-driven Expression Prioritized Integration for Complex Traits) to identify assembled gene-sets that are enriched for genes near associated variants, and to assess whether genes from associated loci were highly expressed in particular tissues or cell types. Using the extended BP locus list based on genome-wide significant loci from this analysis and previously published SNPs that may not have reached genome-wide significance in the current analysis (Supplementary Table 9), we identified five significant (FDR $\leq 5 \%$ ) gene sets: abnormal cardiovascular system physiology, G Alpha 1213 signaling events, embryonic growth retardation, prolonged QT interval, and abnormal vitelline vasculature morphology. We also found that suggestive SBP and DBP associations $\left(P<1 \times 10^{-5}\right)$ were enriched for reconstituted gene-sets at DBP loci (mainly related to developmental pathways), but not at SBP loci (Supplementary Table 22, Supplementary Note). In a final analysis, we assessed Cardio-MetaboChip SNPs at the fine-mapping loci using formaldehyde-assisted isolation of regulatory elements (FAIRE-gen) in lymphoblastoid cell lines ${ }^{35}$. Our results provided support for two SNPs, one of which SNP (rs7961796 at the $T B X 5-T B X 3$ locus) was located in a regulatory site. Although the other SNP (rs3184504 at the $S H 2 B 3$ locus) is a non-synonymous variant, there was also a regulatory site indicated by DNaseI and $\mathrm{H} 3 \mathrm{~K} 4 \mathrm{me} 1$ signatures at the locus, making the SNP a potential regulatory variant (Supplementary Table 23) ${ }^{36}$. Both SNPs were included in the list of $99 \%$ credible SNPs at each locus.

\section{Asian- and African ancestry BP SNP association}

We tested the 66 lead SNPs at the established and novel loci for association with BP in up to 20,875 individuals of South Asian (SAS) ancestry (PROMIS and RACE studies), 9,637 individuals of East Asian (EAS) ancestry (HEXA, HALST, CLHNS, DRAGON, and TUDR studies), and 33,909 individuals of African (AFR) ancestry (COGENT-BP consortium, Jupiter, SPT, Seychelles, GXE, and TANDEM studies). As expected, the effect allele frequencies are very similar across studies of the same ethnicity, but markedly different across different ancestry groups (Supplementary Figure 9). Many associations of 
individual SNPs failed to reach $P<0.05$ for the BP trait with the lower $P$ value

(Supplementary Table 24), which could potentially be due to the much lower statistical power at the sample sizes available, different patterns of LD at each locus across ancestries, variability in allele frequency, or true lack of association in individuals of a given nonEuropean ancestry. The low statistical power for the great majority of SNPs tested is visible considering SNP-by-SNP power calculations using European ancestry effect sizes (Supplementary Table 24). However, concordant directions of allelic effects for both SBP and DBP were observed for 45/66 SNPs in SAS, 36/60 SNPs in EAS, and 42/66 SNPs in AFR samples: the strongest concordance with SAS may not be surprising because South Asians are more closely related to Europeans than are East Asians or Africans. Moreover, strong correlation of effect sizes was observed between EUR samples with SAS, EAS, or AFR samples ( $r=0.55,0.60$, and 0.48 , respectively). A 66-SNP SBP or DBP risk score were significant predictors of SBP and DBP in all samples. A $1 \mathrm{~mm} \mathrm{Hg}$ higher SBP or DBP risk score in EUR samples was associated with a 0.58/0.50 $\mathrm{mm} \mathrm{Hg}$ higher SBP/DBP in SAS samples $\left(\mathrm{SBP} P=1.5 \times 10^{-19}\right.$, DBP $\left.P=3.2 \times 10^{-15}\right), 0.49 / 0.50 \mathrm{~mm} \mathrm{Hg}$ higher SBP/DBP in EAS samples (SBP $P=1.9 \times 10^{-10}$, DBP $P=1.3 \times 10^{-7}$ ), and $0.51 / 0.47 \mathrm{~mm} \mathrm{Hg}$ higher SBP/DBP in AFR samples (SBP $P=2.2 \times 10^{-21}$, DBP $P=6.5 \times 10^{-19}$ ). The attenuation of the genetic risk score estimates in non-European ancestries is presumably due to inclusion of a subset of variants that lack association in the non-European or admixed samples.

We subsequently performed a trans-ethnic meta-analysis of the 66 SNPs in all 64,421 samples across the three non-European ancestries. After correcting for 66 tests, 12/66 SNPs were significantly associated with either SBP or DBP $\left(P<7.6 \times 10^{-4}\right)$, with a correlation of EUR and non-EUR effect estimates of 0.77 for SBP and 0.67 for DBP; the Europeanancestry SBP or DBP risk score was associated with $0.53 / 0.48 \mathrm{~mm} \mathrm{Hg}$ higher BP per predicted $\mathrm{mm} \mathrm{Hg}$ SBP/DBP respectively (SBP $P<6.6 \times 10^{-48}$, DBP $P<1.3 \times 10^{-38}$ ). For 7 of the 12 significant SNPs, no association has previously been reported in genome-wide studies of non-European ancestry. Some heterogeneity of effects was observed between European and non-European effect estimates (Supplementary Table 24). Taken together, these findings suggest that, in aggregate, BP loci identified using data from individuals of EUR ancestry are also predictive of BP in non-EUR samples, but larger non-European sample sizes will be needed to establish precisely which individual SNPs are associated in a given ethnic group.

\section{Impact on hypertensive target organ damage}

Long-term elevated BP causes target organ damage, especially in the heart, kidney, brain, large blood vessels, and the retinal vessels ${ }^{37}$. Consequently, the genetic effect of the 66 SBP and DBP SNPs on end-organ outcomes can be directly tested using the risk score, although some outcomes lacked results for a small number of SNPs. Interestingly, BP risk scores significantly predicted (Supplementary Note) coronary artery disease risk, left ventricular mass and wall thickness, stroke, urinary albumin/creatinine ratio, carotid intima-medial thickness and central retinal artery caliber, but not heart failure or other kidney phenotypes, after accounting for the number of outcomes examined (Table 3). Because outlier effects can affect risk scores, we repeated the risk score analysis removing iteratively SNPs that contributed to statistical heterogeneity (SNP-trait effects relative to SNP-BP effects). 
Heterogeneity was defined based on a multiple testing adjusted significance threshold for Cochran's Q test of homogeneity of effects (Supplementary Note). The risk score analyses restricted to the subset of SNPs showing no heterogeneity of effect revealed essentially identical results, with the exception that urinary albumin/creatinine ratio was no longer significant. The per-SNP results are provided in Supplementary Table 25 and

Supplementary Figures 10. Because large-scale GWAS of non-BP cardiovascular risk factors are available, we examined the BP risk scores as predictors of other cardiovascular risk factors: LDL-cholesterol, HDL-cholesterol, triglycerides, type 2 diabetes, BMI, and height. We observed nominal $(P<0.05)$ associations of the BP risk scores with risk factors, although mostly in the opposite direction to the risk factor-CVD association

(Supplementary Table 26). The failure to demonstrate an effect of BP risk scores on heart failure may reflect limited power from a modest sample size, but the lack of significant effects on renal measures suggests that the epidemiologic relationship of higher BP and worse renal function may not reflect direct consequences of BP elevation.

\section{DISCUSSION}

The study reported here is the largest to date to investigate the genomics of BP in multiple continental ancestries. Our results highlight four major features of inter-individual variation in BP: (1) we identified 66 (17 novel) genome-wide significant loci for SBP and DBP by targeted genotyping in up to 342,415 individuals of European ancestry that cumulatively explain $\sim 3.5 \%$ of the trait; (2) the variants were enriched for cis-regulatory elements, particularly in vascular endothelial cells; (3) the variants had broadly comparable BP effects in South Asians, East Asian and Africans, albeit in smaller sample sizes; and, (4) a 66 SNP risk-score predicted target organ damage in the heart, cerebral vessels, carotid artery and the eye with little evidence for an effect in kidneys. Overall, there was no enrichment of a single genetic pathway in our data; rather, our results are consistent with the effects of BP arising from multiple tissues and organs.

Genetic and molecular analyses of Mendelian syndromes of hypertension and hypotension point largely to a renal origin, involving multiple rare deleterious mutations in proteins that regulate salt-water balance ${ }^{38}$. This is strong support for Guyton's hypothesis that the regulation of sodium excretion by the kidney and its effects on extracellular volume are a prime pathway determining intra-arterial pressure ${ }^{39}$. However, our genetic data from unselected individuals in the general community argues against a single dominant renal effect. The 66 SNPs we identified are not chance effects, but have a global distribution and impact on BP that are consistent as measured by their effects across the many studies metaanalyzed. That they are polymorphic across all continental ancestries argues for their origin and functional effects prior to human continental differentiation.

However several of the 17 novel loci contain strong positional biological candidates, these are described in greater detail in Supplementary Table 27 and the Supplementary Note. The single most common feature we identified was the enrichment of regulatory elements for gene expression in vascular endothelial cells. The broad distribution of these cells across both large and small vessels and across all tissues and organs suggest that functional variation in these cells affects endothelial permeability or vascular smooth muscle cell 
contractility via multiple pathways. These hypotheses will need to be rigorously tested in appropriate models, to assess the contribution of these pathways to BP control, and these pathways could also be targets for systemic anti-hypertensive therapy as they are for the pulmonary circulation ${ }^{42}$.

In summary, these genetic observations may contribute to an improved understanding of BP biology and a re-evaluation of the pathways considered relevant for therapeutic BP control.

\section{ONLINE METHODS}

\section{Cohorts contributing to systolic (SBP) and diastolic blood pressure (DBP) analyses}

Studies contributing to BP association discovery including community- and populationbased collections as well as studies of non-BP traits, analyzed as case and control samples separately. Details on each of the studies including study design and BP measurement are provided in Supplementary Table 1, genotyping information in Supplementary Table 2, and participant characteristics in Supplementary Table 3. All participants provided written informed consent and the studies were approved by local Research Ethics Committees and/or Institutional Review Boards.

\section{European ancestry meta-analysis}

BP was measured using standardized protocols in all studies regardless of whether the primary focus was BP or another trait. We initially analyzed affected and unaffected individuals from samples selected as cases (e.g. type 2 diabetes) or controls, separately. However, because sensitivity analyses did not reveal any significant difference in BP effect size estimates between case and control samples (data not shown), we analyzed all samples combined. When available, the average of two BP measurements was used for association analyses (Supplementary Table 1). If an individual was taking a BP-lowering treatment, the underlying systolic BP (SBP) and diastolic BP (DBP) were estimated by adding $15 \mathrm{mmHg}$ and $10 \mathrm{mmHg}$, respectively, to the measured values, as done in prior analyses.

A meta-analysis of 340,934 individuals of European descent was undertaken in four stages with subsequent validation in an independent cohort. Because stage 1 Cardio-MetaboChip samples included many SNPs selected on the basis of association with BP in earlier GWAS, we performed genomic control using a set of putative null SNPs based on $P>0.10$ in earlier GWAS of SBP and DBP or both. Stage 2 samples with genome-wide genotyping used the entire genome-wide set of SNPs for genomic control given the lack of ascertainment. The study design is summarized in Supplementary Figure 1, and further details are provided in Supplementary Tables 2-5 and the Supplementary Note.

\section{Systematic PubMed search +/- 100kb of each newly discovered index SNP}

All genes with any overlap with a 200kb region centered around each of the 17 newly discovered lead SNPs were identified using the UCSC Genome Browser. A search term was constructed for each gene including the short and long gene name and the terms "blood pressure" and "hypertension" (e.g. for NPPA on chr 1: "NPPA OR natriuretic peptide A 
AND (blood pressure OR hypertension)") and the search results of each search term from

PubMed were individually reviewed.

\section{Trait variance explained}

The trait variance explained by 66 lead SNPs at novel and known loci was evaluated in one study that contributed to the discovery effort: the Atherosclerosis Risk in Communities (ARIC) study. We constructed a linear regression model with all 66 or the subset of 49 known SNPs as a set of predictors of the BP residual after adjustment for covariates of the adjusted treatment-corrected BP phenotype (SBP or DBP). The $\mathrm{r}^{2}$ from the regression model was used as the estimate of trait variance explained.

\section{European ancestry GCTA-COJO analysis}

To identify multiple distinct association signals at any given BP locus, we undertook approximate conditional analyses using a model selection procedure implemented in the GCTA-COJO software package ${ }^{44,45}$. To evaluate the robustness of the GCTA-COJO results to the choice of reference data set, model selection was performed using the LD between variants in separate analyses from two datasets of European descent, both with individuals from the UK with Cardio-MetaboChip genotype data: GoDARTS with 7,006 individuals and WTCCC1-T2D/58BC with 2,947 individuals. Assuming that the LD between SNPs more than $10 \mathrm{Mb}$ away or on different chromosomes is zero, we undertook the GCTA-COJO step wise model selection to select SNPs that were conditionally-independently associated with SBP and DBP, in turn, at a genome-wide significance, given by $P<5 \times 10^{-8}$

(Supplementary Tables 6-8) using the stage 4 combined European GWAS+ CardioMetaboChip meta-analysis.

\section{Conditional analyses in the Women's Genome Health Study (WGHS)}

Multivariable regression modeling was performed for each possible combination of putative independent SNPs from a) model selection implemented in GCTA-COJO and b) a comprehensive manual review of the literature (Supplementary Table 9). Any SNP with $P$ $<5 \times 10^{-8}$ in a previous reported BP GWAS was considered. A total of 46 SNPs were examined (Supplementary Table 10). Genome-wide genotyping data imputed to 1000 Genomes in the WGHS $(\mathrm{N}=23,047)$ were used. Regression modeling was performed in the R statistical language (Supplementary Table 10).

\section{Fine mapping and determination of credible sets of causal SNPs}

The GCTA-COJO and WGHS conditional analyses identified multiple distinct signals of association at multiple loci (Supplementary Tables 6 and 10). Of the 24 loci considered in fine-mapping analyses, 16 had no evidence for the existence of multiple distinct association signals, so it is reasonable to assume that there is a single causal SNP and therefore the credible sets of variants could be constructed using the association summary statistics from the unconditional meta-analyses. However, in the remaining eight loci, where evidence of secondary signals was observed from GCTA-COJO, we performed approximate conditional analyses across the region by conditioning on each index SNP (Supplementary Table 11). By adjusting for the other index SNPs at the locus, we can therefore assume a single variant 
is driving each "conditionally-independent" association signal, and we can construct the 99\% credible set of variants on the basis of the approximate conditional analysis from GCTA-COJO (Supplementary Tables 12-13). At five of the eight loci with multiple distinct signals of association, one index SNP mapped outside of the fine-mapping region, so a credible set could not be constructed.

\section{eQTL analysis: Whole Blood}

NESDA/NTR: Whole blood eQTL analyses were performed in samples from the Netherlands Study of Depression and Anxiety (NESDA) ${ }^{46}$ and the Netherlands Twin Registry (NTR ${ }^{47}$ studies. RNA expression analysis was performed in the statistical software $R$. The residuals resulting from the linear regression analysis of the probe set intensity values onto the covariates sex, age, body mass index $\left(\mathrm{kg} / \mathrm{m}^{2}\right)$, smoking status coded as a categorical covariate, several technical covariates, and three principal components were used. The eQTL effects were detected using a linear mixed model approach, including for each probe set the expression level (normalized, residualized and without the first 50 expression PCs) as dependent variable; the SNP genotype values as fixed effects; and family identifier and zygosity (in the case of twins) as random effects to account for family and twin relations ${ }^{48}$.

The eQTL effects were defined as cis when probe set-SNP pairs were at distance $<1 \mathrm{M}$ base pairs. At a FDR of 0.01 applied genome-wide, not just for candidate SNPs, the $P$ value threshold was $1 \times 10^{-4}$ for the cis-eQTL analysis. For each probe set that displayed a statistically significant association with at least one SNP located within its cis region, we identified the most significantly associated SNP and denoted this as the top cis-eQTL SNP. See Supplementary Note for details.

\section{eQTL analysis: Selected published eQTL datasets}

Lead BP SNP and proxies $\left(r^{2}>0.8\right)$ were searched against a collected database of expression SNP (eSNP) results. The reported eSNP results met criteria for statistical thresholds for association with gene transcript levels as described in the original papers. The non-blood cell tissue eQTLs searched included aortic endothelial cells ${ }^{49}$, left ventricle of the heart ${ }^{50}$, cd14+ monocytes ${ }^{51}$ and the brain ${ }^{52}$. The results are presented in Supplementary

Tables 14-15.

\section{Enrichment analyses: Analysis of cell-specific DNase hypersensitivity sites (DHSs) using an OR method}

The overlap of Cardio-MetaboChip SNPs with DHSs was examined using publicly available data from the Epigenomics Roadmap Project and ENCODE, choosing different cutoffs of Cardio-MetaboChip $P$ values. The DHS mappings were available for 123 mostly adult cells and tissues ${ }^{53}$ (downloaded from The DHS mappings were specified as both "narrow" and "broad" peaks, referring to reduction of the experimental data to peak calls at $0.1 \%$ and $1.0 \%$ FDR thresholds, respectively. Thus, the "narrow" peaks are largely nested within the "broad" peaks. Experimental replicates of the DHS mappings (typically duplicates) were also available for the majority of cells and tissues. 
SNPs from the Cardio-MetaboChip genome-wide scan were first clumped in PLINK in windows of $100 \mathrm{~kb}$ and maximum $\mathrm{r}^{2}=0.1$ among LD relationships from the 1000 Genomes European data. Then, the resulting index SNPs at each $P$ value threshold were tagged with $\mathrm{r}^{2}$ $=0.8$ in windows of $100 \mathrm{~kb}$, again using LD relationships in the 1000 Genomes, restricted to SNPs with MAF > 1\% and also present in the HapMap2 CEU population. A reference set of SNPs was constructed using the same clumping and tagging procedures applied to GWAS catalog SNPs (available at http://www.genome.gov/gwastudies/, accessed 3/13/2013) ${ }^{54}$ with discovery $P<5 \times 10^{-8}$ in European populations. A small number of reference SNPs or their proxies overlapping the BP SNPs or their proxies were excluded. After LD pruning and exclusions, there were a total of 1,196 reference SNPs. For each cell type and $P$ value threshold, the enrichment of SBP or DBP SNPs (or their LD proxies) mapping to DHSs was expressed as an odds ratio (OR) relative to the GWAS catalog reference SNPs (or their LD proxies), using logistic mixed effect models treating the replicate peak determinations as random effects (glmer package in $\mathrm{R}$ ). The significance of the enrichment ORs was derived from the significance of beta coefficients for the main effects in the mixed models (Figure 2, Supplementary Table 16).

\section{Enrichment analyses: Analysis of tissue-specific enrichment of BP variants and H3K4me3 sites}

An analysis to test for significant cell-specific enrichment in the overlap of BP SNPs (or their proxies) with H3K4me3 sites was performed as described in Trynka et al, 2013 ${ }^{55}$. The measure of overlap is a "score" that is constructed by dividing the height of an H3K4me3 ChIP signal in a particular cell by the distance between the nearest test SNP. The significance of the scores (i.e. $P$ value) for all SNPs was determined by a permutation approach that compares the observed scores to scores of SNPs with similar properties to the test SNPs, essentially in terms of LD and proximity to genes (Supplementary Note). The number of permutations determined the number of significant digits in the $P$ values and we conducted 10,000 iterations. Results are shown in Supplementary Table 19.

\section{Enrichment analyses: Analysis of tissue-specific DHSs and chromatin states using GREGOR}

The DNase-seq ENCODE data for all available cell types were downloaded in the processed "narrowPeak" format. The local maxima of the tag density in broad, variable-sized "hotspot" regions of chromatin accessibility were thresholded at FDR $1 \%$ with peaks set to a fixed width of 150bp. Individual cell types were further grouped into 41 broad tissue categories by taking the union of DHSs for all related cell types and replicates. For each GWAS locus, a set of matched control SNPs was selected based on three criteria: 1) number of variants in $\mathrm{LD}\left(\mathrm{r}^{2}>0.7 ; \pm 8\right.$ variants), 2$) \mathrm{MAF}( \pm 1 \%)$, and 3 ) distance to nearest gene ( $\left.\pm 11,655 \mathrm{bp}\right)$. To calculate the distance to the nearest gene, the distance to the 5' flanking gene (start and end position) and to the 3' flanking gene was calculated and the minimum of these 4 values was used. If the SNP fell within the transcribed region of a gene, the distance was 0 . The probability that a set of GWAS loci overlap with a regulatory feature more often than we expect by chance was estimated. 


\section{Enrichment analyses: FAIRE analysis of BP variants in fine-mapping regions in lymphoblastoid cell lines}

FAIRE analysis was performed on a sample of 20 lymphoblastoid cell lines of European origin. All samples were genotyped using the Cardio-MetaboChip genotyping array, and BP SNPs and LD proxies $\left(r^{2}>0.8\right)$ at the fine mapping loci $(N=24$, see Supplementary Table 23) were assessed to identify heterozygous imbalance between non-treated and FAIREtreated chromatin. A paired t-test was used to compare the B allele frequency (BAF) arising from formaldehyde-fixed chromatin sheared by sonication and DNA purified to the BAF when the same chromatin sample underwent FAIRE to enrich for open chromatin. Three hundred and fifty-seven Cardio-MetaboChip BP SNPs were directly genotyped across the fine mapping regions. The Bonferroni-corrected threshold of significance is $P<0.0001$ (0.05/357). The results for SNPs with $P<0.05$ are reported in (Supplementary Table 23). FAIRE results were not available for some SNPs with missing data due to genotype failure or not having $>3$ heterozygous individuals for statistical analysis. Therefore there are no results for three lower frequency BP loci (SLC39A8, CYP17A1-NT5C2 and GNAS-EDN3) and for the second signal at the following loci: MTHFR-NPPB (rs2272803), MECOM (rs2242338) and HFE rs1800562).

\section{Pathway analyses: MAGENTA}

MAGENTA tests for enrichment of gene sets from a precompiled library derived from GO, KEGG, PATHTER, REACTOME, INGENUITY, and BIOCARTA was performed as described by Segré et al, $2010^{56}$. Enrichment of significant gene-wide $P$ values in gene sets is assessed by 1) using LD and distance criteria to define the span of each gene, 2) selecting the smallest $P$ value among SNPs mapping to the gene span, and 3) adjusting this $P$ value using a regression method that accounts for the number of SNPs, the LD, etc. In the second step, MAGENTA examines the distribution of these adjusted $P$ values and defines thresholds for the $75 \%$ ile and the $95 \%$ ile. In the third step, MAGENTA calculates an enrichment for each gene set by comparing the number of genes in the gene set with $P$ value less than either the 75th or 95th \%ile to the number of genes in the gene set with $P$ value greater than either the 75th or 95th \%ile, and then comparing this quotient to the same quotient among genes not in the gene set. This gene-set quotient is assigned a $P$ value based on reference to a hypergeometric distribution. The results based on our analyses are indicated in Supplementary Table 21.

\section{Pathway analyses: DEPICT}

We applied the DEPICT ${ }^{57}$ analysis separately on genome-wide significant loci from the overall blood pressure (BP) Cardio-MetaboChip analysis including published blood pressure loci (see Supplementary Table 22). SNPs at the HFE and BAT2-BAT5 loci (rs1799945, rs1800562, rs2187668, rs805303, rs9268977) could not be mapped. As a secondary analysis, we additionally included associated loci $\left(P<1 \times 10^{-5}\right)$ from the Cardio-MetaboChip stage 4 combined meta-analyses of SBP and the DBP. DEPICT assigned genes to associated regions if they overlapped or resided within associated LD blocks with $\mathrm{r}^{2}>0.5$ to a given associated SNP. 


\section{Literature review for genes at the newly discovered loci}

Recognizing that the most significantly associated SNP at a locus may not be located in the causal gene and that the functional consequences of a SNP often extends beyond $100 \mathrm{~kb}$, we conducted a literature review of genes in extended regions around newly discovered BP index SNPs. The genes for this extensive review were identified by DEPICT

(Supplementary Table 22).

\section{Non-European meta-analysis}

To assess the association of the 66 significant loci from the European ancestry meta-analysis in non-European ethnicities, we obtained lookup results for the 66 index SNPs for participants of South-Asian ancestry ( 8 datasets, total $\mathrm{N}=20,875)$, East-Asian ancestry ( 5 datasets, total $\mathrm{N}=9,637$ ), and African- and African-American ancestry ( 6 datasets, total $\mathrm{N}=$ $33,909)$. The association analyses were all conducted with the same covariates (age, age ${ }^{2}$, sex, BMI) and treatment correction $(+15 / 10 \mathrm{~mm} \mathrm{Hg}$ in the presence of any hypertensive medication) as the association analyses for the discovery effort in Europeans. Tests for heterogeneity across effect estimates in European, South Asian, East Asian and African derived samples were performed using GWAMA ${ }^{58}$.

\section{Genetic risk score and cardiovascular outcomes}

The gtx package for the $\mathrm{R}$ statistical programming language was used to estimate the effect of the SNP-risk score on the response variable in a regression model ${ }^{59}$.

\section{Supplementary Material}

Refer to Web version on PubMed Central for supplementary material.

\section{Authors}

Georg B. Ehret ${ }^{\# 1,2}$, Teresa Ferreira ${ }^{\# 3}$, Daniel I. Chasman 4,5 , Anne U. Jackson ${ }^{6,7}$, Ellen M. Schmidt ${ }^{8}$, Toby Johnson ${ }^{9,10}$, Gudmar Thorleifsson ${ }^{11}$, Jian'an Luan ${ }^{12}$, Lousie A. Donnelly ${ }^{13}$, Stavroula Kanoni ${ }^{14}$, Ann-Kristin Petersen ${ }^{15}$, Vasyl Pihur ${ }^{1}$, Rona J. Strawbridge ${ }^{16,17}$, Dmitry Shungin ${ }^{18,19,20}$, Maria F. Hughes ${ }^{21}$, Osorio Meirelles $^{22}$, Marika Kaakinen ${ }^{23}$, Nabila Bouatia-Najii24,25, Kati Kristiansson ${ }^{26,27}$, Sonia Shah ${ }^{28}$, Marcus E. Kleber ${ }^{29}$, Xiuqing Guo ${ }^{30}$, Leo-Pekka Lyytikäinen ${ }^{31,32}$, Cristiano Fava ${ }^{33,34}$, Niclas Eriksson ${ }^{35}$, Ilja M. Nolte ${ }^{36}$, Patrik K. Magnusson ${ }^{37}$, Elias L. Salfati ${ }^{38}$, Loukianos S. Rallidis ${ }^{39}$, Elizabeth Theusch ${ }^{40}$, Andrew J.P. Smith ${ }^{41}$, Lasse Folkersen ${ }^{16}$, Kate Witkowska ${ }^{9,42}$, Tune H. Pers ${ }^{43,44,45,46,47}$, Roby Joehanes $^{48}$, Stuart K. Kim ${ }^{49}$, Lazaros Lataniotis ${ }^{14}$, Rick Jansen ${ }^{50}$, Andrew D. Johnson ${ }^{48,51}$, Helen Warren 9,42 , Young Jin Kim², Wei Zhao ${ }^{53}$, Ying Wu ${ }^{54}$, Bamidele O. Tayo ${ }^{55}$, Murielle Bochud ${ }^{56}$, CHARGE-EchoGen consortium ${ }^{57}$, CHARGE-HF consortium ${ }^{57}$, Wellcome Trust Case Control Consortium ${ }^{57}$, Devin Absher ${ }^{58}$, Linda S. Adair ${ }^{59}$, Najaf Amin ${ }^{60}$, Dan E. Arking ${ }^{1}$, Tomas Axelsson ${ }^{61}$, Damiano Baldassarre ${ }^{62,63}$, Beverley Balkau ${ }^{64}$, Stefania Bandinelli65, Michael R. Barnes $^{14,42}$, Inês Barroso $66,67,68$, Stephen Bevan ${ }^{69}$, Joshua C. Bis ${ }^{70}$, Gyda Bjornsdottir $^{11}$, Michael Boehnke ${ }^{6,7}$, Eric Boerwinkle ${ }^{71}$, Lori L. Bonnycastle ${ }^{72}$, Dorret 
I. Boomsma 73 , Stefan R. Bornstein 74 , Morris J. Brown ${ }^{75}$, Michel Burnier ${ }^{76}$, Claudia P. Cabrera ${ }^{9,42}$, John C. Chambers ${ }^{77,78,79}$, I-Shou Chang ${ }^{80}$, Ching-Yu Cheng ${ }^{81,82,83}$, Peter S. Chines ${ }^{72}$, Ren-Hua Chung ${ }^{84}$, Francis S. Collins ${ }^{72}$, John M. Connel| ${ }^{85}$, Angela Döring ${ }^{86,87}$, Jean Dallongeville ${ }^{88}$, John Danesh ${ }^{89,66,90}$, Ulf de Faire ${ }^{91}$, Graciela Delgado ${ }^{29}$, Anna F. Dominiczak ${ }^{92}$, Alex S.F. Doney ${ }^{13}$, Fotios Drenos ${ }^{41}$, Sarah Edkins ${ }^{66}$, John D. Eicher ${ }^{48,51}$, Roberto Elosua ${ }^{93}$, Stefan Enroth ${ }^{94,95}$, Jeanette Erdmann ${ }^{96,97}$, Per Eriksson ${ }^{16}$, Tonu Esko ${ }^{98,99,100}$, Evangelos Evangelou ${ }^{77,101}$, Alun Evans ${ }^{21}$, Tove Fall ${ }^{102}$, Martin Farrall 3,103 , Janine F. Felix ${ }^{104}$, Jean Ferrières ${ }^{105}$, Luigi Ferrucci ${ }^{106}$, Myriam Fornage ${ }^{107}$, Terrence Forrester ${ }^{108}$, Nora Franceschini ${ }^{109}$, Oscar H. Franco Duran ${ }^{104}$, Anders Franco-Cereceda ${ }^{101}$, Ross M. Fraser ${ }^{111,112}$, Santhi K. Ganesh ${ }^{113}$, He Gao ${ }^{77}$, Karl Gertow ${ }^{16,17}$, Francesco Gianfagna ${ }^{114,115}$, Bruna Gigante ${ }^{91}$, Franco Giulianini ${ }^{4}$, Anuj Goel ${ }^{3,103}$, Alison H. Goodall ${ }^{116,117}$, Mark O. Goodarzi ${ }^{118}$, Mathias Gorski19,120, Jürgen Gräßler $^{121}$, Christopher Groves ${ }^{122}$, Vilmundur Gudnason ${ }^{123,124}$, Ulf Gyllensten ${ }^{94,95}$, Göran Hallmans ${ }^{18}$, Anna-Liisa Hartikainen ${ }^{125,126}$, Maija Hassinen ${ }^{127}$, Aki S. Havulinna $^{26}$, Caroline Hayward ${ }^{128}$, Serge Hercberg ${ }^{129}$, Karl-Heinz Herzig ${ }^{130,131,132}$, Andrew A. Hicks ${ }^{133}$, Aroon D. Hingorani28, Joel N. Hirschhorn43,44,45,134, Albert Hofman ${ }^{104,135}$, Jostein Holmen ${ }^{136}$, Oddgeir Lingaas Holmen ${ }^{136,137}$, Jouke-Jan Hottenga ${ }^{73}$, Phil Howard ${ }^{41}$, Chao A. Hsiung ${ }^{84}$, Steven C. Hunt ${ }^{138,139}$, M. Arfan Ikram $^{104,140,141}$, Thomas Illig 142,143,144, Carlos Iribarren ${ }^{145}$, Richard A. Jensen ${ }^{71,146}$, Mika Kähönen ${ }^{147}$, Hyun Kang ${ }^{6,7}$, Sekar Kathiresan 148,149,150,45,151, Brendan J. Keating ${ }^{152,153}$, Kay-Tee Khaw ${ }^{154}$, Yun Kyoung Kim ${ }^{52}$, Eric Kim ${ }^{155}$, Mika Kivimaki $^{28}$, Norman Klopp ${ }^{142,143}$, Genovefa Kolovou ${ }^{156}$, Pirjo Komulainen ${ }^{127}$, Jaspal S. Kooner $157,78,79$, Gulum Kosova ${ }^{149,148,100}$, Ronald M. Krauss ${ }^{158}$, Diana Kuh $^{159}$, Zoltan Kutalik ${ }^{160,161}$, Johanna Kuusisto ${ }^{162}$, Kirsti Kvaløy ${ }^{136}$, Timo A Lakka $^{163,127,164}$, Nanette R. Lee ${ }^{165,166}$, I-Te Lee ${ }^{167,168}$, Wen-Jane Lee ${ }^{169}$, Daniel Levy ${ }^{48,170}$, Xiaohui Li ${ }^{30}$, Kae-Woei Liang 171,172, Honghuang Lin ${ }^{173,48}$, Li Lin², Jaana Lindström ${ }^{26}$, Stéphane Lobbens ${ }^{174,175,176}$, Satu Männistö ${ }^{26}$, Gabriele Müller $^{177}$, Martina Müller-Nurasyid ${ }^{15,178,179}$, François Mach ${ }^{2}$, Hugh S. Markus ${ }^{180}$, Eirini Marouli ${ }^{14,181}$, Mark I. McCarthy ${ }^{122}$, Colin A. McKenzie ${ }^{108}$, Pierre Meneton ${ }^{182}$, Cristina Menni ${ }^{183}$, Andres Metspalu ${ }^{98}$, Vladan Mijatovic ${ }^{184}$, Leena Moilanen ${ }^{185,186}$, May E. Montasser ${ }^{187}$, Andrew D. Morris ${ }^{13}$, Alanna C. Morrison ${ }^{188}$, Antonella Mulas $^{189}$, Ramaiah Nagaraja ${ }^{22}$, Narisu Narisu ${ }^{72}$, Kjell Nikus ${ }^{190,191}$, Christopher J. O'Donnell ${ }^{192,48,151}$, Paul F. O'Reilly ${ }^{193}$, Ken K. Ong ${ }^{12}$, Fred Paccaud ${ }^{56}$, Cameron D. Palmer 194,195,45, Afshin Parsa ${ }^{187}$, Nancy L. Pedersen 37 , Brenda W.

Penninx ${ }^{196,197,198}$, Markus Perola ${ }^{26,27,98}$, Annette Peters ${ }^{87}$, Neil Poulter ${ }^{199}$, Peter P. Pramstaller133,200,201, Bruce M. Psaty70,202,203,204, Thomas Quertermous ${ }^{38}$, Dabeeru C. Rao 205 , Asif Rasheed ${ }^{206}$, N William N.W.R. Rayner ${ }^{122,3,66}$, Frida Renström ${ }^{19,207,18}$, Rainer Rettig 208 , Kenneth M. Rice ${ }^{209}$, Robert Roberts ${ }^{210,211}$, Lynda M. Rose ${ }^{4}$, Jacques Rossouw ${ }^{212}$, Nilesh J. Samani ${ }^{116,213}$, Serena Sanna ${ }^{189}$, Jouko Saramies ${ }^{214}$, Heribert Schunkert ${ }^{215,216,217,218}$, Sylvain Sebert $219,131,164$, Wayne H.-H. Sheu ${ }^{167,168,220}$, Young-Ah Shin ${ }^{52}$, Xueling Sim ${ }^{6,7,221}$, Johannes H. Smit ${ }^{196}$, Albert V. Smith ${ }^{123,124}$, Maria X. Sosa1, Tim D. Spector ${ }^{183}$, Alena Stančáková222, Alice Stanton ${ }^{223}$, Kathleen E. Stirrups ${ }^{14,224}$, Heather M. Stringham ${ }^{6,7}$, Johan Sundstrom ${ }^{61}$, Amy J. Swift ${ }^{72}$, Ann-Christine Syvänen ${ }^{61}$, E- 
Shyong Tai225,82,221, Toshiko Tanaka106, Kirill V. Tarasov²26, Alexander Teumer227, Unnur Thorsteinsdottir ${ }^{11,124}$, Martin D. Tobin ${ }^{228}$, Elena Tremoli62,63, Andre G. Uitterlinden ${ }^{104,229}$, Matti Uusitupa ${ }^{230,231}$, Ahmad Vaez ${ }^{36,232}$, Dhananjay Vaidya ${ }^{233}$, Cornelia M. van Duijn ${ }^{104,234}$, Erik P.A. van Iperen ${ }^{235,236}$, Ramachandran S. Vasan $48,237,238$, Germaine C. Verwoert ${ }^{104}$, Jarmo Virtamo ${ }^{26}$, Veronique Vitart ${ }^{128}$, Benjamin F. Voight ${ }^{45,239}$, Peter Vollenweider ${ }^{240}$, Aline Wagner ${ }^{241}$, Louise V. Wain $^{228}$, Nicholas J. Wareham ${ }^{12}$, Hugh Watkins ${ }^{3,103}$, Alan B. Weder ${ }^{242}$, Harm-Jan Westra $^{243}$, Rainford Wilks ${ }^{244}$, Tom Wilsgaard245,246, James F. Wilson ${ }^{111,128}$, Tien Y. Wong ${ }^{81,82,83}$, Tsun-Po Yang ${ }^{14,247}$, Jie Yao ${ }^{30}$, Loic Yengo ${ }^{174,175,176}$, Weihua Zhang ${ }^{77,78}$, Jing Hua Zhao ${ }^{12}$, Xiaofeng Zhu ${ }^{248}$, Pascal Bovet ${ }^{249,56}$, Richard S. Cooper ${ }^{55}$, Karen L. Mohlke ${ }^{54}$, Danish Saleheen ${ }^{250,206}$, Jong-Young Lee ${ }^{52}$, Paul Elliott ${ }^{77,251}$, Hinco J. Gierman ${ }^{49,252}$, Cristen J. Willer ${ }^{8,253,254}$, Lude Franke255, G Kees Hovingh ${ }^{256}$, Kent D. Taylor ${ }^{30}$, George Dedoussis ${ }^{181}$, Peter Sever ${ }^{199}$, Andrew Wong ${ }^{159}$, Lars Lind ${ }^{61}$, Themistocles L. Assimes ${ }^{38}$, Inger Njølstad ${ }^{245,246}$, Peter EH. Schwarz $^{74}$, Claudia Langenberg ${ }^{12}$, Harold Snieder ${ }^{36}$, Mark J. Caulfield ${ }^{9,42}$, Olle Melander $^{33}$, Markku Laakso ${ }^{162}$, Juha Saltevo ${ }^{257}$, Rainer Rauramaa ${ }^{127,164}$, Jaakko Tuomilehto 26,258,259,260, Erik Ingelsson ${ }^{102,3}$, Terho Lehtimäki ${ }^{31,32}$, Kristian Hveem $^{136}$, Walter Palmas ${ }^{261}$, Winfried März ${ }^{262,263}$, Meena Kumari ${ }^{28}$, Veikko Salomaa ${ }^{26}$, Yii-Der I. Chen ${ }^{30}$, Jerome I. Rotter ${ }^{30}$, Philippe Froguel174,175,176,23, Marjo-Riitta Jarvelin $219,131,264,251$, Edward G. Lakatta ${ }^{226}$, Kari Kuulasmaa ${ }^{26}$, Paul W. Franks ${ }^{19,207,18}$, Anders Hamsten ${ }^{16,17}$, H.-Erich Wichmann ${ }^{86,179,265}$, Colin N.A. Palmer $^{13}$, Kari Stefansson ${ }^{11,124}$, Paul M Ridker ${ }^{4,5}$, Ruth J.F. Loos ${ }^{12,266,267,}$

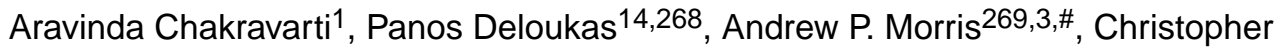
Newton-Cheh $148,149,45,100, \#$, and Patricia B. Munroe $9,42, \#$

\section{Affiliations}

${ }^{1}$ Center for Complex Disease Genomics, McKusick-Nathans Institute of Genetic Medicine, Johns Hopkins University School of Medicine, Baltimore, MD 21205, USA

${ }^{2}$ Cardiology, Department of Medicine, Geneva University Hospital, Rue GabriellePerret-Gentil 4, 1211 Geneva 14, Switzerland ${ }^{3}$ Wellcome Trust Centre for Human Genetics, University of Oxford, Oxford, OX3 7BN, UK ${ }^{4}$ Division of Preventive Medicine, Brigham and Women's Hospital, 900 Commonwealth Ave. East, Boston, MA 02215, USA ${ }^{5}$ Harvard Medical School, Boston, MA 02115, USA ${ }^{6}$ Department of Biostatistics, University of Michigan, Ann Arbor, MI 48109, USA ${ }^{7}$ Center for Statistical Genetics, University of Michigan, Ann Arbor, MI 48109, USA 8 Department of Computational Medicine and Bioinformatics, University of Michigan, Ann Arbor, MI 48109, USA ${ }^{9}$ Clinical Pharmacology, William Harvey Research Institute, Queen Mary University of London, London, EC1M 6BQ, UK 10 GlaxoSmithKline, Gunnels Wood Road, Stevenage SG1 2NY, UK ${ }^{11}$ deCODE Genetics/Amgen, Inc., Reykjavik, Iceland ${ }^{12}$ MRC Epidemiology Unit, University of Cambridge School of Clinical Medicine, Institute of Metabolic Science, Cambridge Biomedical Campus, Cambridge, CB2 0QQ, UK ${ }^{13}$ Medical Research Institute, University of Dundee, Ninewells Hospital and Medical School, Dundee, DD1 9SY, UK ${ }^{14}$ William Harvey Research Institute, Barts and The London School of Medicine and Dentistry, Queen Mary University of London, London, UK ${ }^{15}$ Institute of Genetic 
Epidemiology, Helmholtz Zentrum München, Neuherberg 85764, Germany ${ }^{16}$ Cardiovascular Research Unit, Center for Molecular Medicine L8:03, Department of Medicine, Karolinska Institutet, 17176 Stockholm, Sweden ${ }^{17}$ Center for Molecular Medicine, Karolinska University Hospital Solna, Stockholm, Sweden ${ }^{18}$ Department of Public Health and Clinical Medicine, Umeå University, Sweden ${ }^{19}$ Department of Clinical Sciences, Genetic and Molecular Epidemiology Unit, Skåne University Hospital Malmö, SE-205 02 Malmö, Sweden ${ }^{20}$ Department of Odontology, Umeå University, Sweden ${ }^{21}$ Centre of Excellence for Public Health, Queens University Belfast, Grosvenor Road, Belfast BT126JP, UK ${ }^{22}$ Laboratory of Genetics, Intramural Research Program, National Institute on Aging, National Institutes of Health, Baltimore, Maryland 21224, USA ${ }^{23}$ Department of Genomics of Common Disease, School of Public Health, Imperial College London, Hammersmith Hospital, London, UK 24 INSERM UMR970, Paris Cardiovascular Research Center PARCC, 56 rue Leblanc, 75015 Paris, France ${ }^{25}$ University Paris-Descartes, Sorbonne Paris Cité, 12 rue de l'Ecole de medicine, F-75006 Paris, France ${ }^{26}$ National Institute for Health and Welfare, FI-00271 Helsinki, Finland ${ }^{27}$ Institute for Molecular Medicine Finland FIMM, University of Helsinki, 00290 Helsinki, Finland ${ }^{28}$ Genetic Epidemiology Group, Dept. Epidemiology and Public Health, UCL, London, WC1E 6BT, UK ${ }^{29}$ Vth Department of Medicine, Medical Faculty Mannheim, Heidelberg University, Theodor-Kutzer-Ufer 1-3, 68167 Mannheim, Germany ${ }^{30}$ Institute for Translational Genomics and Population Sciences, Los Angeles Biomedical Research Institute at Harbor-UCLA Medical Center, 1124 W. Carson Street, Torrance, CA 90502, USA 31 Department of Clinical Chemistry, Fimlab Laboratories, Tampere 33520, Finland 32 Department of Clinical Chemistry, University of Tampere School of Medicine, Tampere 33014, Finland ${ }^{33}$ University of Lund, Dept Internal Medicine, Malmo, SE 20502, Sweden ${ }^{34}$ University of Verona, Dept of Internal Medicine, Verona, Italy $37134{ }^{35}$ Uppsala University, Uppsala Clinical Research Center, SE-75185 Uppsala, Sweden ${ }^{36}$ Department of Epidemiology, University of Groningen, University Medical Center Groningen, Hanzeplein 1, 9713 GZ Groningen, The Netherlands ${ }^{37}$ Dept of Medical Epidemiology and Biostatistics, Karolinska Institutet, Box 281, SE-171 77 Stockholm, Sweden ${ }^{38}$ Department of Medicine, Stanford University School of Medicine, Stanford, CA 94305, USA ${ }^{39}$ Second Department of Cardiology, Attikon Hospital, School of Medicine, University of Athens, Athens, Greece ${ }^{40}$ Children's Hospital Oakland Research Institute, Oakland, CA 94609, USA ${ }^{41}$ Department of Cardiovascular Genetics, Institute of Cardiovascular Sciences, University College London, London WC1E 6JF, UK ${ }^{42}$ NIHR Barts Cardiovascular Biomedical Research Unit, Queen Mary University of London, London, EC1M 6BQ, UK ${ }^{43}$ Division of Endocrinology, Boston Children's Hospital, Boston, MA 02115, USA ${ }^{44}$ Center for Basic and Translational Obesity Research, Boston Children's Hospital, Boston, MA 02115, USA 45 Program in Medical and Population Genetics, Broad Institute, 7 Cambridge Center, Cambridge, MA 02142, USA 46 Novo Nordisk Foundation Centre for Basic Metabolic Research, Section of Metabolic, Genetics, Faculty of Health and Medical Sciences, University of Copenhagen, Copenhagen, 2100, Denmark 47 Department of Epidemiology Research, Statens Serum Institut, 2300, Copenhagen, 
Denmark ${ }^{48}$ National Heart, Lung and Blood Institute's Framingham Heart Study, Framingham, MA 01702, USA ${ }^{49}$ Dept. Dev. Bio. And Genetics, Stanford University Medical Center, Stanford, CA 94305, USA 50 Department of Psychiatry, VU University Medical Center, Amsterdam, The Netherlands ${ }^{51}$ National Heart, Lung and Blood Institute, Cardiovascular Epidemiology and Human Genomics Branch, Bethesda, MD 20814, USA 52 Center for Genome Science, National Institute of Health, Osong Health Technology Administration Complex, Chungcheongbuk-do, Republic of Korea ${ }^{53}$ Division of Translational Medicine and Human Genetics, Department of Medicine, University of Pennyslvania, USA ${ }^{54}$ Department of Genetics, University of North Carolina, Chapel Hill, NC 27599, USA ${ }^{55}$ Department of Preventive Medicine and Epidemiology, Loyola University Chicago Stritch School of Medicine, Maywood, IL, 60153, USA ${ }^{56}$ Institute of Social and Preventive Medicine (IUMSP), Centre Hospitalier Universitaire Vaudois and University of Lausanne, Route de la Corniche 10, 1010 Lausanne, Switzerland ${ }^{58}$ HudsonAlpha Institute for Biotechnology, Huntsville, AL 35086, USA ${ }^{59}$ Department of Nutrition, University of North Carolina, Chapel Hill, NC 27599, USA ${ }^{60}$ Genetic Epidemiology Unit, Department of Epidemiology, Erasmus MC, Rotterdam, 3015CN, The Netherlands ${ }^{61}$ Uppsala University, Department of Medical Sciences, SE-75185 Uppsala, Sweden ${ }^{62}$ Dipartimento di Scienze Farmacologiche e Biomolecolari, Università di Milano, Milan, Italy ${ }^{63}$ Centro Cardiologico Monzino, IRCCS, Milan, Italy 64 INSERM Centre for Research in Epidemiology and Population Health, U1018, Villejuif, France University Paris-Sud, URMS 1018, Villejuif, France ${ }^{65}$ Geriatric Unit, Azienda Sanitaria Firenze (ASF), Florence, Italy ${ }^{66}$ Wellcome Trust Sanger Institute, Wellcome Trust Genome Campus, CB10 1SA, Hinxton, UK ${ }^{67}$ University of Cambridge Metabolic Research Laboratories, Level 4, Institute of Metabolic Science Box 289 Addenbrookes Hospital Cambridge CB2 OQQ, UK ${ }^{68}$ NIHR Cambridge Biomedical Research Centre, Level 4, Institute of Metabolic Science Box 289 Addenbrookes Hospital Cambridge CB2 OQQ, UK ${ }^{69}$ School of Life Science, University of Lincoln, Joseph Banks Laboratories, Lincoln LN6 7DL, UK 70 Cardiovascular Health Research Unit, Department of Medicine, University of Washington, Seattle, WA 98101, USA ${ }^{71}$ Human Genetics Center, School of Public Health, University of Texas Health Science Center at Houston, 1200 Pressler St., Suite 453E, Houston, TX 77030, USA 72 Medical Genomics and Metabolic Genetics Branch, National Human Genome Research Institute, NIH, Bethesda, MD 20892, USA ${ }^{73}$ Department of Biological Psychology, VU University, Amsterdam, The Netherlands ${ }^{74}$ Dept of Medicine III, University of Dresden, Medical Faculty Carl Gustav Carus, Fetscherstrasse 74, 01307 Dresden, Germany ${ }^{75}$ The Barts Heart Centre, William Harvey Research Institute, Queen Mary University of London, London EC1M 6BQ, UK ${ }^{76}$ Nephrology, Centre Hospitalier Universitaire Vaudois and University of Lausanne, Bugnon 17, 1005 Lausanne, Switzerland ${ }^{77}$ Department of Epidemiology and Biostatistics, School of Public Health, Imperial College London, Norfolk Place, London W2 1PG, UK ${ }^{78}$ Department of Cardiology, Ealing Hospital NHS Trust, Uxbridge Road, Southall, Middlesex UB1 3EU, UK ${ }^{79}$ Imperial College Healthcare NHS Trust, London, UK ${ }^{80}$ National Institute of Cancer Research, 
National Health Research Institutes. 35 Keyan Rd., Zhunan Town, Miaoli County 350, Taiwan ${ }^{81}$ Singapore Eye Research Institute, Singapore National Eye Centre, Singapore 168751, Singapore ${ }^{82}$ Duke-NUS Graduate Medical School Singapore, Singapore 169857, Singapore ${ }^{83}$ Department of Ophthalmology, National University of Singapore and National University Health System, Singapore $119228{ }^{84}$ Division of Biostatistics and Bioinformatics, Institute of Population Health Sciences, National Health Research Institutes. 35 Keyan Rd., Zhunan Town, Miaoli County 350, Taiwan 85 University of Dundee, Ninewells Hospital and Medical School, Dundee, DD1 9SY, UK ${ }^{86}$ Institute of Epidemiology I, Helmholtz Zentrum München, Neuherberg 85764, Germany ${ }^{87}$ Institute of Epidemiology II, Helmholtz Zentrum München, Neuherberg 85764, Germany 88 UMR744 Inserm-Lille2-Institut Pasteur Lille, France 89 Department of Public Health and Primary Care, University of Cambridge, Cambridge CB1 8RN, UK ${ }^{90}$ NIHR Blood and Transplant Research Unit in Donor Health and Genomics, Department of Public Health and Primary Care, University of Cambridge, Cambridge CB1 8RN, UK ${ }^{91}$ Division of Cardiovascular Epidemiology, Institute of Environmental Medicine, Karolinska Institutet, Stockholm, Sweden ${ }^{92}$ BHF Glasgow Cardiovascular Research Centre, Institute of Cardiovascular and Medical Sciences, University of Glasgow, 126 University Place, Glasgow, G12 8QT, UK ${ }^{93}$ Cardiovascular Epidemiology and Genetics. IMIM (Institut Hospital del Mar d'Investigacions Mèdiques), Barcelona, Spain ${ }^{94}$ Department of Immunology, Genetics and Pathology, University of Uppsala, Box 815, Biomerical center, 75108 Uppsala, Sweden ${ }^{95}$ Science for Life Laboratory, University of Uppsala, Box 815, Biomerical center, 75108 Uppsala, Sweden ${ }^{96}$ Institut für Integrative und Experimentelle Genomik, Universiät zu Lübeck, RatzeburgerAllee 160, 23538 Lübeck, Germany ${ }^{97}$ Deutsches Zentrum für Herz-Kreislauf-Forschung (DZHK), partner site Hamburg, Kiel, Lübeck, Universität zu Lübeck, Lübeck, Germany 98 Estonian Genome Center, University of Tartu, Tartu, 51010, Estonia ${ }^{99}$ Divisions of Endocrinology/Children's Hospital, Boston, MA 02115, USA ${ }^{100}$ Broad Institute of Harvard and MIT, Cambridge, MA 02139 USA ${ }^{101}$ Department of Hygiene and Epidemiology, University of loannina Medical School, loannina, 45110, Greece ${ }^{102}$ Department of Medical Sciences, Molecular Epidemiology and Science for Life Laboratory, Uppsala University, Uppsala, Sweden ${ }^{103}$ Division of Cardiovascular Medicine, Radcliffe Department of Medicine, University of Oxford, Oxford, OX3 9DU, UK ${ }^{104}$ Department of Epidemiology, Erasmus MC, University Medical Center Rotterdam, P.O.Box 2040, 3000 CA Rotterdam, The Netherlands ${ }^{105}$ Toulouse University School of Medicine, Rangueil University Hospital, INSERM UMR1027, Toulouse, France ${ }^{106}$ Translational Gerontology Branch, National Institute on Aging, Baltimore MD, USA ${ }^{107}$ Institute of Molecular Medicine, University of Texas Health Science Center at Houston, TX, USA 108 Tropical Metabolism Research Unit, Tropical Medicine Research Institute, University of the West Indies, Mona, Kingston 7, Jamaica ${ }^{109}$ Department of Epidemiology, University of North Carolina, Chapel Hill, NC 27599, USA ${ }^{110}$ Cardiothoracic Surgery Unit, Department of Molecular Medicine and Surgery, Karolinska Institutet, 17176 Stockholm, Sweden ${ }^{111}$ Institute for Population Health Sciences and Informatics, University of Edinburgh, Teviot 
Place, Edinburgh, EH8 9AG, Scotland ${ }^{112}$ Synpromics Ltd, 9 Bioquarter, Little France Road, Edinburgh, EH16 4UX, Scotland ${ }^{113}$ University of Michigan Medical School, 7220 MSRB III, Ann Arbor MI 48109, USA ${ }^{114}$ EPIMED Research Centre Epidemiology and Preventive Medicine, Department of Clinical and Experimental Medicine, University of Insubria, Varese, Italy ${ }^{115}$ Department of Epidemiology and Prevention, IRCCS Istituto Neurologico Mediterraneo NEUROMED, 86077 Pozzilli, Italy ${ }^{116}$ Department of Cardiovascular Sciences, University of Leicester, Glenfield Hospital, Leicester LE3 9QP, UK ${ }^{117}$ National Institute for Health Research Leicester Cardiovascular Biomedical Research Unit, Glenfield Hospital, Leicester LE3 9QP, UK ${ }^{118}$ Division of Endocrinology, Diabetes and Metabolism, Cedars-Sinai Medical Center, Los Angeles, CA 90048, USA ${ }^{119}$ Department of Genetic Epidemiology, Institute of Epidemiology and Preventive Medicine, University of Regensburg, FranzJosef-Strauss-Allee 11, 93053 Regensburg, Germany ${ }^{120}$ Department of Nephrology, University Hospital Regensburg, Franz-Josef-Strauss-Allee 11, 93053 Regensburg, Germany ${ }^{121}$ Department of Medicine III, Division Pathobiochemistry, Technische Universität Dresden, Dresden, Germany ${ }^{122}$ Oxford Centre for Diabetes, Endocrinology and Metabolism, University of Oxford, Oxford, UK ${ }^{123}$ Icelandic Heart Association, Kopavogur, Iceland ${ }^{124}$ Faculty of Medicine, University of Iceland, Reykjavik, Iceland ${ }^{125}$ Institute of Clinical Medicine/Obstetrics and Gynaecology, University of Oulu, Oulu, Finland ${ }^{126}$ Medical Research Center, Oulu University Hospital, Oulu, Finland ${ }^{127}$ Kuopio Research Institute of Exercise Medicine, Kuopio, Finland ${ }^{128}$ Institute of Genetics and Molecular Medicine, Western General Hospital, Edinburgh, EH4 2XU Scotland, UK ${ }^{129}$ UREN, INSERM U557, INRA U1125, CNAM, $\mathrm{SMBH}$, Sorbonne Paris Cité, Université Paris 13, Bobigny, France ${ }^{130}$ Institute of Biomedicine, University of Oulu, Medical Research Center Oulu and Oulu University Hospital, Finland ${ }^{131}$ Biocenter Oulu, P.O.Box 5000, Aapistie 5A, FI-90014 University of Oulu, Finland ${ }^{132}$ Department of Gastroenterology and Metabolism, Poznan University of Medical Sciences, Poznan, Poland ${ }^{133}$ Center for Biomedicine, European Academy Bozen/Bolzano (EURAC), Bolzano, 39100, Italy - affiliated institute of the University of Lübeck, Germany ${ }^{134}$ Department of Genetics, Harvard Medical School, Boston, 02115, USA ${ }^{135}$ Department of Epidemiology, Harvard T.H. Chan School of Public Health, Boston, MA, USA ${ }^{136}$ HUNT Research Centre, Department of Public Health and General Practice, Norwegian University of Science and Technology, 7600 Levanger, Norway ${ }^{137}$ St. Olav Hospital, Trondheim University Hospital, Trondheim, Norway ${ }^{138}$ Cardiovascular Genetics Division, University of Utah School of Medicine, Salt Lake City, Utah, USA ${ }^{139}$ Department of Genetic Medicine, Weill Cornell Medical College Qatar, Doha, Qatar ${ }^{140}$ Department of Radiology, Erasmus MC, The Netherlands ${ }^{141}$ Department of Neurology, Erasmus MC, University Medical Center Rotterdam, P.O.Box 2040, 3000 CA Rotterdam, The Netherlands ${ }^{142}$ Research Unit of Molecular Epidemiology, Helmholtz Zentrum München, Neuherberg 85764, Germany ${ }^{143}$ Hannover Unified Biobank, Hannover Medical School, Hannover 30625, Germany ${ }^{144}$ Hannover Medical School, Institute for Human Genetics, Carl-Neuberg-Strasse 1, 30625 Hanover, Germany ${ }^{145}$ Kaiser Permanente, Division of Research, Oakland, CA 94612, USA ${ }^{146}$ Department of 
Medicine, University of Washington, Seattle, Washington 98101, USA 147

Department of Clinical Physiology, Tampere University Hospital, Tampere 33521, Finland ${ }^{148}$ Center for Human Genetic Research, Massachusetts General Hospital, Boston, MA 02114, USA ${ }^{149}$ Cardiovascular Research Center, Massachusetts General Hospital, Boston, MA 02114, USA ${ }^{150}$ Department of Medicine, Harvard Medical School, Boston, MA, USA ${ }^{151}$ Cardiology Division, Department of Medicine, Massachusetts General Hospital ${ }^{152}$ Division of Transplantation, Department of Surgery, University of Pennsylvania, PA 19104 USA ${ }^{153}$ Department of Pediatrics, University of Pennsylvania, Philadelphia, PA, USA ${ }^{154}$ Department of Public Health and Primary Care, Institute of Public Health, University of Cambridge, Cambridge CB2 2SR, UK ${ }^{155}$ Institute for Translational Genomics and Population Sciences, Department of Pediatrics, LABioMed at Harbor-UCLA Medical Center, 1124 W. Carson Street, Torrance, CA 90502, USA 156 1st Cardiology Department, Onassis Cardiac Surgery Center 356, Sygrou Ave, Athens, Greece ${ }^{157}$ National Heart and Lung Institute, Imperial College London, Hammersmith Hospital Campus, Ducane Road, London W12 0NN, UK ${ }^{158}$ Department of Medicine, Children's Hospital Oakland Research Institute, Oakland, CA 94609, USA ${ }^{159}$ MRC Unit for Lifelong Health and Ageing at UCL, London, WC1B 5JU, UK ${ }^{160}$ Department of Medical Genetics, University of Lausanne, Lausanne, Switzerland ${ }^{161}$ Swiss Institute of Bioinformatics, Lausanne, Switzerland ${ }^{162}$ Department of Medicine, University of Eastern Finland and Kuopio University Hospital, 70210 Kuopio, Finland ${ }^{163}$ Institute of Biomedicine/Physiology, University of Eastern Finland, Kuopio Campus, Finland 164 Department of Clinical Physiology and Nuclear Medicine, Kuopio University Hospital, Kuopio, Finland ${ }^{165}$ Office of Population Studies Foundation Inc., Talamban, Cebu City, 6000, Philippines ${ }^{166}$ Department of Anthropology, Sociology, and History, University of San Carlos, Talamban, Cebu City, 6000, Philippines ${ }^{167}$ Division of Endocrine and Metabolism, Department of Internal Medicine, Chichung Veterans General Hospital, Taichung 40705, Taiwan ${ }^{168}$ School of Medicine, National Yang-Ming University, Taipei, Taiwan ${ }^{169}$ Department of Medical Research, Taichung Veterans General Hospital, Taichung 407, Taiwan ${ }^{170}$ Population Sciences Branch, National Heart Lung, and Blood Institute, National Institutes of Health, Bethesda, MD, USA ${ }^{171}$ Cardiovascular Center, Taichung Veterans General Hospital, Taichung, 40705, Taiwan ${ }^{172}$ Institute of Clinical Medicine, National Yang Ming University School of Medicine, Taipei 112, Taiwan ${ }^{173}$ Section of Computational Biomedicine, Department of Medicine, Boston University School of Medicine, Boston, 02446 MA, USA ${ }^{174}$ European Genomic Institute for Diabetes (EGID), FR 3508 Lille, France ${ }^{175}$ Centre National de la Recherche Scientifique (CNRS) UMR 8199, Lille Pasteur Institute, 1 rue du Prof Calmette, 59019 Lille Cedex, France 176 Lille 2 University, Lille, France ${ }^{177}$ Center for Evidence-based Healthcare, University of Dresden, Medical Faculty Carl Gustav Carus, Fetscherstrasse 74, 01307 Dresden, Germany ${ }^{178}$ Department of Medicine I, University Hospital Grosshadern, Ludwig-Maximilians University, Munich, Germany ${ }^{179}$ Institute of Medical Informatics, Biometry and Epidemiology, Chair of Epidemiology, LudwigMaximilians-Universität, München 81377, Germany ${ }^{180}$ Neurology Unit, University of 
Cambridge, R3, Box 83, Cambridge Biomedical Campus, Cambridge, Cb2 0QQ, UK 181 Department of Dietetics-Nutrition, Harokopio University, 70 El. Venizelou Str, Athens, Greece ${ }^{182}$ INSERM U1142 LIMICS, UMR_S 1142 Sorbonne Universités, UPMC Université Paris 06, Université Paris 13, Paris, France ${ }^{183}$ Department of Twin Research and Genetic Epidemiology, King's College London, London, UK 184 Department of Life and Reproduction Sciences, University of Verona, Strada le Grazie 8, 37134 Verona, Italy ${ }^{185}$ Department of Medicine, Kuopio University Hospital, Kuopio, Finland ${ }^{186}$ Unit of General Practice, Oulu University Hospital, Oulu, Finland ${ }^{187}$ Department of Medicine, Program for Personalized and Genomic Medicine, University of Maryland, School of Medicine, Baltimore, Maryland 21201, USA ${ }^{188}$ Department of Epidemiology, Human Genetics and Environmental Sciences, School of Public Health, University of Texas Health Science Center at Houston, 1200 Pressler St., Suite 453E, Houston, TX 77030, USA ${ }^{189}$ Istituto di Ricerca Genetica e Biomedica (IRGB), Consiglio Nazionale delle Ricerche, c/o Cittadella Universitaria di Monseratto, Monserrato, Cagliari 09042, Italy 190 Department of Cardiology, School of Medicine, University of Tampere, Tampere 33014, Finland ${ }^{191}$ School of Medicine, University of Tampere, Tampere 33014, Finland 192 National Heart, Lung and Blood Institute, Division of Intramural Research, Bethesda, MD, USA ${ }^{193}$ Institute of Psychiatry, Psychology and Neuroscience, King's College London, London SE5 8AF, UK ${ }^{194}$ Divisions of Endocrinology, Children's Hospital Boston, Massachusetts 02115, USA 195 Genetics and Program in Genomics, Children's Hospital Boston, Massachusetts 02115, USA 196 Department of Psychiatry, EMGO Institute, Neuroscience Campus, VU University Medical Centre, Van der Boechorststraat 7, 1081 BT Amsterdam, The Netherlands ${ }^{197}$ Department of Psychiatry, University of Groningen, University Medical Center Groningen, Hanzeplein 1, 9713 GZ Groningen, The Netherlands ${ }^{198}$ Department of Psychiatry, Leiden University Medical Centre, P.O. Box 9600, 2300 RC Leiden, The Netherlands ${ }^{199}$ International Centre for Circulatory Health, Imperial College London, W2 1PG, UK 200 Department of Neurology, General Central Hospital, Bolzano, 39100, Italy ${ }^{201}$ Department of Neurology, University of Lübeck, Lübeck, Germany 202 Department of Epidemiology, University of Washington, Seattle, WA, USA 203 Department of Health Services, University of Washington, Seattle, WA 204 Group Health Research Institute, Group Health Cooperative, Seattle, WA 205 Division of Biostatistics, Washington University School of Medicine, Saint Louis, MO, 63110, USA ${ }^{206}$ Center for Non-Communicable Diseases, Karachi, Pakistan 207 Department of Nutrition, Harvard School of Public Health, Boston, MA, USA 208 Institute of Physiology, University Medicine Greifswald, Greifswald, Germany ${ }^{209}$ Department of Biostatistics, University of Washington, Seattle, WA, USA 210 University of Ottawa Heart Institute, Cardiovascular Research Methods Centre Ontario, Canada ${ }^{211}$ Ruddy Canadian Cardiovascular Genetics Centre, Ontario, Canada 212 National Heart, Lung, and Blood Institute, 6701 Rockledge Ave., Bethesda, MD 20892, USA ${ }^{213}$ Leicester NIHR Biomedical Research Unit in Cardiovascular Disease, Glenfield Hospital, Leicester LE3 9QP, UK ${ }^{214}$ South Karelia Central Hospital, Lappeenranta, Finland ${ }^{215}$ Deutsches Herzzentrum 
München, Germany ${ }^{216}$ Technische Universität München, Germany ${ }^{217}$ Deutsches Zentrum für Herz-Kreislauf-Forschung (DZHK), München, Germany ${ }^{218}$ Munich Heart Alliance, Germany ${ }^{219}$ Center For Life-course Health Research, P.O.Box 5000, FI-90014 University of Oulu, Finland ${ }^{220}$ College of Medicine, National Defense Medical Center, Taipei, Taiwan ${ }^{221}$ Saw Swee Hock School of Public Health, National University of Singapore and National University Health System, Singapore $117597^{222}$ University of Eastern Finland and Kuopio University Hospital, 70210 Kuopio, Finland ${ }^{223}$ Conway Institute of Biomolecular and Biomedical Research, University College Dublin, Dublin 4, Ireland ${ }^{224}$ Department of Haematology, University of Cambridge, Cambridge, UK ${ }^{225}$ Department of Medicine, National University of Singapore and National University Health System, Singapore 119228, Singapore ${ }^{226}$ Laboratory of Cardiovascular Science, Intramural Research Program, National Institute on Aging, National Institutes of Health, Baltimore, Maryland, 21224, USA 227 Institute for Community Medicine, University Medicine Greifswald, Greifswald, Germany ${ }^{228}$ Department of Health Sciences, University of Leicester, University Rd, Leicester LE1 7RH, UK ${ }^{229}$ Department of internal medicine, Erasmus MC, Rotterdam, 3000CA, The Netherlands ${ }^{230}$ Department of Public Health and Clinical Nutrition, University of Eastern Finland, Finland ${ }^{231}$ Research Unit, Kuopio University Hospital, Kuopio, Finland ${ }^{232}$ Research Institute for Primordial Prevention of Non-communicable Disease, Isfahan University of Medical Sciences, Isfahan, Iran 233 Johns Hopkins Medical Institutions, 1830 East Monument St., Baltimore, MD 21287, USA ${ }^{234}$ Centre of Medical Systems Biology (CMSB 1-2), NGI Erasmus Medical Center, Rotterdam, The Netherlands 235 Department of Clinical Epidemiology, Biostatistics and Bioinformatics, Academic Medical Center, Amsterdam, The Netherlands ${ }^{236}$ Durrer Center for Cardiogenetic Research, ICIN-Netherlands Heart Institute, Utrecht, The Netherlands ${ }^{237}$ Section of Preventive medicine, Department of Medicine, Boston University School of Medicine, Boston, 02446 MA, USA 238 Cardiology, Department of Medicine, Boston University School of Medicine, Boston, 02446 MA, USA ${ }^{239}$ Department of Pharmacology, University of Pennsylvania Perelman School of Medicine, Philadelphia, Pennsylvania, USA 240 Department of Internal medicine, University Hospital Lausanne, Lausanne, Switzerland ${ }^{241}$ Department of Epidemiology and Public Health, EA3430, University of Strasbourg, Strasbourg, France 242 Division of Cardiovascular Medicine, Department of Internal Medicine, University of Michigan Medical School, Ann Arbor, MI, USA ${ }^{243}$ University Medical Center Groningen, University of Groningen, Groningen, 9700RB, The Netherlands ${ }^{244}$ Epidemiology Research Unit, Tropical Medicine Research Institute, University of the West Indies, Mona, Kingston 7, Jamaica ${ }^{245}$ Department of Community Medicine, Faculty of Health Sciences, University of Troms $\varnothing$, Troms $\varnothing$, Norway ${ }^{246}$ Department of Clinical Medicine, Faculty of Health Sciences, University of Tromsø, Troms $\varnothing$, Norway 247 MRC Cancer Unit, University of Cambridge, Cambridge, UK ${ }^{248}$ Department of Epidemiology and Biostatistics, School of Medicine, Case Western Reserve University, Cleveland, $\mathrm{OH}, 44106$, USA ${ }^{249}$ Ministry of Health, Victoria, Republic of Seychelles ${ }^{250}$ Department of Biostatistics and Epidemiology, University of 
Pennsylvania, USA ${ }^{251}$ MRC-PHE Centre for Environment and Health, School of Public Health, Imperial College London, Norfolk Place, London W2 1PG, UK 252 Enterprise Informatics, Illumina Inc., Santa Clara CA, 95050, USA ${ }^{253}$ Department of Internal Medicine, Division of Cardiovascular Medicine, University of Michigan, Ann Arbor, MI 48109, USA 254 Department of Human Genetics, University of Michigan, Ann Arbor, Michigan, USA ${ }^{255}$ Department of Genetics, University of Groningen, University Medical Centre Groningen, Groningen, 9711, The Netherlands ${ }^{256}$ Dept Vascular Medicine, Academic Medical Center, Amsterdam, The Netherlands 257 Department of Medicine, Central Finland Health Care District, Jyväskylä, Finland 258 Dasman Diabetes Institute, Dasman, 15462 Kuwait 259 Saudi Diabetes Research Group, King Abdulaziz University, 21589 Jeddah, Saudi Arabia ${ }^{260}$ Centre for Vascular Prevention, Danube-University Krems, 3500 Krems, Austria 261 Department of Medicine, Columbia University, 622 West 168th St., New York, NY 10032, USA 262 Synlab Academy, Synlab Services GmbH, P5, 7, 68161 Mannheim, Germany ${ }^{263}$ Clinical Institute of Medical and Chemical Laboratory Diagnostics, Medical University of Graz, 8036 Graz, Austria ${ }^{264}$ Unit of Primary Care, Oulu University Hospital, Kajaanintie 50, P.O.Box 20, FI-90220 Oulu, 90029 OYS, Finland 265 Grosshadern, Klinikum, München 81377, Germany 266 The Charles Bronfman Institute for Personalized Medicine, The Icahn School of Medicine at Mount Sinai, New York, NY 10029, USA ${ }^{267}$ Mindich Child health Development Institute, The Icahn School of Medicine at Mount Sinai, New York, NY 10029, USA 268 Princess Al-Jawhara Al-Brahim Centre of Excellence in Research of Hereditary Disorders (PACER-HD), King Abdulaziz University, Jeddah 21589, Saudi Arabia 269 Department of Biostatistics, University of Liverpool, Liverpool L69 3GA, UK

\section{ACKNOWLEDGEMENTS}

We thank all the study participants of this study for their contributions. Detailed acknowledgment of funding sources is provided in the Supplementary Note.

\section{AUTHOR CONTRIBUTIONS}

Analysis group

Design of secondary analyses: G.B.E., T.Ferreira, T.J., A.P.M., P.B.M., C.N.-C. Computation of secondary analyses: G.B.E., T.Ferreira, T.J., A.P.M., P.B.M., C.N.-C. Paper writing: A.C., G.B.E., T.Ferreira, T.J., A.P.M., P.B.M., C.N.-C. Study management: P.B.M., C.N.-C.

Cardio-MetaboChip or new GWAS

WGHS: Study phenotyping: P.M.R., D.I.C., L.M.R. Genotyping or analysis: P.M.R., D.I.C., L.M.R., F.Giulianini Study PI: P.M.R.

JUPITER: Study phenotyping: P.M.R., D.I.C., L.M.R. Genotyping or analysis: D.I.C., L.M.R., F.Giulianini Study PI: P.M.R., D.I.C. 
deCODE: Study phenotyping: G.B. Genotyping or analysis: G.T. Study PI: K.S., U.T.

GoDARTS: Study phenotyping: C.N.A.P., L.A.D., A.D.M., A.S.F.D. Genotyping or analysis: C.N.A.P., L.A.D., A.D.M., M.I.M., C.G., N.W.W.R.R. Study PI: C.N.A.P., A.D.M.

KORA F3/F4: Study phenotyping: A.D., H.Schunkert, J.E. Genotyping or analysis: A.-K.P., M.M.-N., N.K., T.I. Study PI: H.-E.W., A.Peters

GLACIER: Study phenotyping: F.R., G.H. Genotyping or analysis: P.W.F., D.Shungin, I.B., S.Edkins, F.R. Study PI: P.W.F.

B58C: Genotyping or analysis: S.Kanoni, K.E.S., Wellcome Trust Case Control Consortium, E.M., T.Ferreira, T.J. Study PI: P.D.

MORGAM: Study phenotyping: K.Kuulasmaa, F.Gianfagna, A.Wagner, J.Dallongeville Genotyping or analysis: M.F.H., F.Gianfagna Study PI: J.V., J.F., A.E.

SardiNIA: Study phenotyping: E.G.L. Genotyping or analysis: E.G.L., O.Meirelles, S.Sanna, R.N., A.Mulas, K.V.T.

NFBC1986: Study phenotyping: M.R.J., S.Sebert, K.H.H., A.L.H. Genotyping or analysis: M.Kaakinen, A.L.H. Study PI: M.R.J.

DESIR: Genotyping or analysis: N.B.-N., L.Y., S.L. Study PI: P.F., N.B.-N., B.B.

DILGOM: Study phenotyping: S.M. Genotyping or analysis: K.Kristiansson, M.P., A.S.H. Study PI: V.S.

IMPROVE: Study phenotyping: D.B. Genotyping or analysis: R.J.S., K.G. Study PI:

A.Hamsten, E.Tremoli

HyperGEN: Study phenotyping: S.C.H., D.C.R. Genotyping or analysis: A.C., V.P., G.B.E. Study PI: S.C.H.

FENLAND (MetaboChip): Study phenotyping: R.J.F.L., J.a.L., N.J.W., K.K.O. Genotyping or analysis: R.J.F.L., J.a.L., N.J.W., K.K.O. Study PI: N.J.W.

Whitehall II: Study phenotyping: M.Kumari Genotyping or analysis: M.Kumari, S.Shah, C.L. Study PI: A.Hingorani, M.Kivimaki

LURIC: Genotyping or analysis: M.E.K., G.Delgado Study PI: W.M.

MESA: Study phenotyping: W.P. Genotyping or analysis: W.P., X.G., J.Y., V.D., K.D.T., J.I.R., Y.-D.C. Study PI: W.P.

HUNT2: Study phenotyping: K.Kvaløy, J.H., O.L.H. Genotyping or analysis: A.U.J. Study PI: K.H.

FINCAVAS: Genotyping or analysis: T.L., L.-P.L., K.N., M.Kähönen Study PI: T.L., M.Kähönen 
GenNet: Study phenotyping: R.S.C., A.B.W. Genotyping or analysis: A.C., V.P., M.X.S., D.E.A., G.B.E. Study PI: A.C., R.S.C., A.B.W.

SCARFSHEEP: Study phenotyping: B.G. Genotyping or analysis: R.J.S. Study PI:

A.Hamsten, U.d.F.

DPS: Study phenotyping: J.L. Genotyping or analysis: A.U.J., P.S.C. Study PI: J.T., M.U.

DR's EXTRA: Study phenotyping: P.K. Genotyping or analysis: A.U.J., M.H. Study PI:

R.Rauramaa, T.A.L.

FIN-D2D 2007: Genotyping or analysis: A.U.J., L.L.B. Study PI: J.Saltevo, L.M.

METSIM: Study phenotyping: H.M.S. Genotyping or analysis: A.U.J., A.Stančáková Study PI: M.L., J.K.

MDC-CVA: Study phenotyping: O.Melander Genotyping or analysis: O.Melander, C.F.

Study PI: O.Melander

BRIGHT: Study phenotyping: A.F.D., M.J.B., N.J.S., J.M.C. Genotyping or analysis: T.J., P.B.M. Study PI: M.J.C., A.F.D., M.J.B., N.J.S., J.M.C., P.B.M.

NESDA: Study phenotyping: J.H.S. Genotyping or analysis: H.Snieder, I.M.N. Study PI: B.W.P.

EPIC (MetaboChip): Study phenotyping: R.J.F.L., J.a.L., N.J.W. Genotyping or analysis: J.a.L., N.J.W. Study PI: N.J.W., K.-T.K.

ELY: Study phenotyping: C.L., J.a.L., N.J.W. Genotyping or analysis: C.L., J.a.L., N.J.W. Study PI: N.J.W.

DIAGEN: Study phenotyping: J.G., G.M. Genotyping or analysis: A.U.J., G.M. Study PI: P.E.S., S.R.B.

GOSH: Study phenotyping: P.K.M., N.L.P. Genotyping or analysis: E.I., P.K.M., N.L.P., T.Fall Study PI: E.I.

Tromsø: Study phenotyping: T.W. Genotyping or analysis: A.U.J., A.J.S., N. Study PI: I.N.

ADVANCE: Study phenotyping: T.L.A., C.I. Genotyping or analysis: T.L.A., E.L.S., T.Q. Study PI: T.L.A., T.Q., C.I.

ULSAM: Study phenotyping: E.I., J.Sundstrom Genotyping or analysis: E.I., N.E., J.Sundstrom, A.-C.S. Study PI: J.Sundstrom

PIVUS: Study phenotyping: L.Lind, J.Sundstrom Genotyping or analysis: L.Lind, N.E., J.Sundstrom, T.A. Study PI: L.Lind, J.Sundstrom

MRC NSHD: Study phenotyping: D.K. Genotyping or analysis: A.Wong, J.a.L., D.K., K.K.O. Study PI: D.K. 
ASCOT: Study phenotyping: A.Stanton, N.P. Genotyping or analysis: T.J., M.J.C., P.B.M. Study PI: P.S., M.J.C.

THISEAS: Genotyping or analysis: L.S.R., S.Kanoni, E.M., G.Kolovou Study PI:

G.Dedoussis, P.D.

PARC: Study phenotyping: R.M.K. Genotyping or analysis: K.D.T., E.Theusch, J.I.R., X.L., M.O.G., Y.D.I.C. Study PI: R.M.K.

AMC-PAS: Genotyping or analysis: G.K.H., P.D. Study PI: G.K.H.

CARDIOGENICS: Genotyping or analysis: S.Kanoni, A.H.G. Study PI: P.D., A.H.G., J.E., N.J.S., H.Schunkert

Secondary analyses

Allele-specific FAIRE: Design of secondary analysis: A.J.P.S. Computation of secondary analysis: A.J.P.S., F.D., P.H.

ASAP eQTL: Design of secondary analysis: A.F.C. Computation of secondary analysis:

L.Folkersen, P.Eriksson

CARDIOGENICS eQTL: Computation of secondary analysis: L.Lataniotis

CM design: P.B.M., C.N.-C., T.J., B.F.V.

Comprehensive literature review: Design of secondary analysis: P.B.M. Computation of secondary analysis: K.W., P.B.M.

DEPICT: Design of secondary analysis: L.Franke, T.H.P., J.N.H. Computation of secondary analysis: T.H.P.

DHS and methylation analysis by tissue:Design of secondary analysis: C.J.W. Computation of secondary analysis: E.M.S.

DHS and methylation by cell-line: Design of secondary analysis: D.I.C. Computation of secondary analysis: D.I.C., F.Giulianini

FHS eSNP: Design of secondary analysis: R.Joehanes Computation of secondary analysis:

R.Joehanes

ICBP SC: C.N.-C., M.J.C., P.B.M., A.C., K.M.R., P.-O'R., W.P., D.L., M.D.T., B.M.P., A.D.J., P.Elliott, C.M.v.D., D.I.C., A.V.S., M.Bochud, L.V.W., H.Snieder, G.B.E.

Kidney eQTL: Computation of secondary analysis: H.J.G., S.K.K.

MAGENTA: Design of secondary analysis: D.I.C. Computation of secondary analysis:

D.I.C.

Miscellaneous: Computation of secondary analysis: H.Warren 
MuTHER eQTL: Design of secondary analysis: P.D. Computation of secondary analysis: L.Lataniotis, T.-P.Y.

NESDA eQTL: Design of secondary analysis: R.Jansen Computation of secondary analysis: R.Jansen, A.V.

NTR eQTL: Design of secondary analysis: R.Jansen Computation of secondary analysis: R.Jansen, J.-J.H. Study PI: D.I.B.

eQTL, EGCUT:Design of secondary analysis: A.Metspalu Computation of secondary analysis: T.E., A.Metspalu

eQTL, Groningen:Design of secondary analysis: L.Franke Computation of secondary analysis: H.J.W., L.Franke

Public eSNP and methylation: Design of secondary analysis: A.D.J., J.D.E. Computation of secondary analysis: A.D.J., J.D.E.

PubMed search: Design of secondary analysis: G.B.E. Computation of secondary analysis: G.B.E., L.Lin

WGHS conditional: Design of secondary analysis: D.I.C. Computation of secondary analysis: D.I.C., F.Giulianini, L.M.R.

Lookup of Cardio-MetaboChip variants

HEXA: Genotyping or analysis: Y.J.K., Y.K.K., Y.-A.S. Study PI: J.-Y.L.

RACe: Study phenotyping: D.Saleheen, W.Zhao, A.R., A.R. Genotyping or analysis: W.Zhao, A.R., A.R. Study PI: D.Saleheen

HALST: Study phenotyping: C.A.H. Genotyping or analysis: J.I.R., Y.-D.C., C.A.H., R.H.C., I.-S.C. Study PI: C.A.H.

CLHNS: Study phenotyping: N.R.L., L.S.A. Genotyping or analysis: Y.W., N.R.L., L.S.A. Study PI: K.L.M., L.S.A.

GxE/Spanish Town: Study phenotyping: B.O.T., C.A.M., R.W. Genotyping or analysis: C.D.P. Study PI: R.S.C., C.A.M., R.W., T.Forrester, J.N.H.

DRAGON: Study phenotyping: W.-J.L., W.H.-H.S., K.-W.L., I-Te Lee Genotyping or analysis: J.I.R., Y.-D.C., E.K., D.A., K.D.T., X.G. Study PI: W.H.-H.S.

SEY: Study phenotyping: P.B. Genotyping or analysis: M.Bochud, G.B.E., F.M. Study PI: P.B., M.Bochud, M.Burnier, F.P.

TUDR: Study phenotyping: W.H.-H.S., I-Te Lee, W.-J.L. Genotyping or analysis: J.I.R., Y.D.C., E.K., K.D.T., X.G. Study PI: W.H.-H.S. 
TANDEM: Study phenotyping: P.B., M.Bochud Genotyping or analysis: G.B.E., F.M. Study PI: P.B., M.Bochud, M.Burnier, F.P.

Imputed genotypes

FHS: Study phenotyping: D.L. Genotyping or analysis: D.L. Study PI: D.L.

ARIC: Study phenotyping: E.B. Genotyping or analysis: G.B.E., E.B., A.C.M., A.C., S.K.G. Study PI: E.B., A.C.

RS: Genotyping or analysis: G.C.V., A.G.U. Study PI: A.Hofman, A.G.U., O.H.F.D.

CoLaus: Study phenotyping: P.V. Genotyping or analysis: Z.K. Study PI: P.V.

NFBC1966: Study phenotyping: M.R.J. Genotyping or analysis: P.O.R. Study PI: M.R.J.

SHIP: Study phenotyping: R.Rettig Genotyping or analysis: A.T.

CHS: Study phenotyping: B.M.P. Genotyping or analysis: K.M.R. Study PI: B.M.P.

EPIC (GWAS): Study phenotyping: N.J.W., R.J.F.L., J.a.L. Genotyping or analysis: N.J.W., J.H.Z., J.a.L. Study PI: N.J.W., K.-T.K.

SU.VI.MAX: Study phenotyping: S.H. Genotyping or analysis: S.H., P.M. Study PI: P.M.

Amish: Genotyping or analysis: M.E.M. Study PI: A.Parsa

FENLAND (GWAS): Study phenotyping: N.J.W., J.a.L., R.J.F.L., K.K.O. Genotyping or analysis: N.J.W., J.a.L., R.J.F.L., K.K.O. Study PI: N.J.W.

DGI: Study phenotyping: C.N.C. Genotyping or analysis: C.N.C., G.Kosova Study PI:

C.N.C.

ERF (EUROSPAN): Genotyping or analysis: N.A. Study PI: C.M.v.D.

MIGEN: Study phenotyping: S.Kathiresan, R.E. Genotyping or analysis: S.Kathiresan, R.E. Design of secondary analysis: S.Kathiresan, R.E.

MICROS: Study phenotyping: P.P.P. Genotyping or analysis: A.A.H. Study PI: A.A.H., P.P.P.

FUSION: Genotyping or analysis: A.U.J. Study PI: M.Boehnke, F.S.C., K.L.M., J.Saramies

TwinsUK: Genotyping or analysis: C.M. Study PI: T.D.S.

PROCARDIS: Genotyping or analysis: M.Farrall, A.G. Study PI: M.Farrall

BLSA: Study phenotyping: L.Ferrucci Genotyping or analysis: T.T. Study PI: L.Ferrucci

ORCADES: Study phenotyping: J.F.W. Study PI: J.F.W. 
Croatia-Vis: Genotyping or analysis: V.V., C.H. Study PI: V.V., C.H.

NSPHS: Genotyping or analysis: S.Enroth Study PI: U.G.

InCHIANTI: Genotyping or analysis: T.T. Study PI: S.Bandinelli

AGES Reykjavik: Study phenotyping: V.G. Genotyping or analysis: A.V.S. Study PI: V.G.

Lookup

CARDIoGRAMplusC4D: Genotyping or analysis: P.D. Study PI: J.Danesh, H.Schunkert, T.L.A., J.E., S.Kathiresan, R.Roberts, N.J.S., P.D.

CHARGE cIMT: Genotyping or analysis: C.O'D., J.C.B.

CHARGE EYE: Genotyping or analysis: T.Y.W., X.S., R.A.J. Study PI: T.Y.W.

CHARGE-HF consortium: Study phenotyping: R.S.V., J.F.F. Genotyping or analysis: H.L., J.F.F. Study PI: R.S.V.

CKDGen: Genotyping or analysis: M.G., V.M.

COGENT: Study phenotyping: N.F., J.R. Genotyping or analysis: N.F., X.Z., B.J.K., B.O.T., J.R.

EchoGen consortium: Study phenotyping: R.S.V., J.F.F. Genotyping or analysis: H.L., J.F.F. Study PI: R.S.V.

KidneyGen Consortium: Study phenotyping: J.C.C., J.S.K., P.Elliott Genotyping or analysis: W.Zhang, J.C.C., J.S.K. Study PI: J.C.C., J.S.K.

MetaStroke: Genotyping or analysis: S.Bevan, H.S.M.

NeuroCHARGE: Genotyping or analysis: M.Fornage, M.A.I. Study PI: M.A.I.

PROMIS: Study phenotyping: D.Saleheen, W.Zhao, J.Danesh Genotyping or analysis:

W.Zhao Study PI: D.Saleheen

SEED: Study phenotyping: T.Y.W., C.-Y.C. Genotyping or analysis: E.-S.T, C.-Y.C., C.-Y.C. Study PI: C.-Y.C., T.Y.W.

UK Biobank: BP group leaders: Mark Caulfield, P.Elliott Genotyping or analysis: M.R.B., H.Warren, Claudia Cabrera, Evangelos Evangelou, He Gao.

\section{REFERENCES}

1. Johnson T, et al. Blood Pressure Loci Identified with a Gene-Centric Array. The American Journal of Human Genetics. 2011; 89:1-13.

2. Newton-Cheh C, et al. Association of common variants in NPPA and NPPB with circulating natriuretic peptides and blood pressure. Nature genetics. 2009; 41:348-53. [PubMed: 19219041] 
3. Franceschini N, et al. Genome-wide association analysis of blood-pressure traits in Africanancestry individuals reveals common associated genes in African and non-African populations. Am J Hum Genet. 2013; 93:545-54. [PubMed: 23972371]

4. Ganesh SK, et al. Effects of long-term averaging of quantitative blood pressure traits on the detection of genetic associations. Am J Hum Genet. 2014; 95:49-65. [PubMed: 24975945]

5. Ehret GB, et al. Genetic variants in novel pathways influence blood pressure and cardiovascular disease risk. Nature. 2011; 478:103-109. [PubMed: 21909115]

6. Wain LV, et al. Genome-wide association study identifies six new loci influencing pulse pressure and mean arterial pressure. Nat Genet. 2011; 43:1005-11. [PubMed: 21909110]

7. Newton-Cheh $\mathrm{C}$, et al. Genome-wide association study identifies eight loci associated with blood pressure. Nat Genet. 2009; 41:666-76. [PubMed: 19430483]

8. Simino J, et al. Gene-age interactions in blood pressure regulation: a large-scale investigation with the CHARGE, Global BPgen, and ICBP Consortia. Am J Hum Genet. 2014; 95:24-38. [PubMed: 24954895]

9. Tragante V, et al. Gene-centric meta-analysis in 87,736 individuals of European ancestry identifies multiple blood-pressure-related loci. Am J Hum Genet. 2014; 94:349-60. [PubMed: 24560520]

10. Wang Y, et al. From the Cover: Whole-genome association study identifies STK39 as a hypertension susceptibility gene. Proc Natl Acad Sci U S A. 2009; 106:226-31. [PubMed: 19114657]

11. Kato N, et al. Meta-analysis of genome-wide association studies identifies common variants associated with blood pressure variation in east Asians. Nature genetics. 2011; 43:531-8. [PubMed: 21572416]

12. Padmanabhan S, et al. Genome-wide association study of blood pressure extremes identifies variant near UMOD associated with hypertension. PLoS genetics. 2010; 6:e1001177. [PubMed: 21082022]

13. Miall WE, Oldham PD. The hereditary factor in arterial blood-pressure. Br Med J. 1963; 1:75-80. [PubMed: 13935402]

14. Levy D, et al. Framingham Heart Study 100K Project: genome-wide associations for blood pressure and arterial stiffness. BMC Med Genet. 2007; 8(Suppl 1):S3. [PubMed: 17903302]

15. Teslovich TM, et al. Biological, clinical and population relevance of 95 loci for blood lipids. Nature. 2010; 466:707-13. [PubMed: 20686565]

16. Voight BF, et al. The metabochip, a custom genotyping array for genetic studies of metabolic, cardiovascular, and anthropometric traits. PLoS Genet. 2012; 8:e1002793. [PubMed: 22876189]

17. Tobin MD, Sheehan NA, Scurrah KJ, Burton PR. Adjusting for treatment effects in studies of quantitative traits: antihypertensive therapy and systolic blood pressure. Stat Med. 2005; 24:291135. [PubMed: 16152135]

18. Locke AE, et al. Genetic studies of body mass index yield new insights for obesity biology. Nature. 2015; 518:197-206. [PubMed: 25673413]

19. Shungin D, et al. New genetic loci link adipose and insulin biology to body fat distribution. Nature. 2015; 518:187-96. [PubMed: 25673412]

20. Sudlow C, et al. UK biobank: an open access resource for identifying the causes of a wide range of complex diseases of middle and old age. PLoS Med. 2015; 12:e1001779. [PubMed: 25826379]

21. Levy D, et al. Genome-wide association study of blood pressure and hypertension. Nat Genet. 2009; 41:677-87. [PubMed: 19430479]

22. Yang J, et al. Conditional and joint multiple-SNP analysis of GWAS summary statistics identifies additional variants influencing complex traits. Nat Genet. 2012; 44:369-75. S1-3. [PubMed: 22426310]

23. Yang J, Lee SH, Goddard ME, Visscher PM. GCTA: a tool for genome-wide complex trait analysis. Am J Hum Genet. 2011; 88:76-82. [PubMed: 21167468]

24. Kimber CH, et al. TCF7L2 in the Go-DARTS study: evidence for a gene dose effect on both diabetes susceptibility and control of glucose levels. Diabetologia. 2007; 50:1186-91. [PubMed: 17429603] 
25. Erdmann J, et al. Dysfunctional nitric oxide signalling increases risk of myocardial infarction. Nature. 2013; 504:432-6. [PubMed: 24213632]

26. Hirata $Y$, et al. Mechanisms of adrenomedullin-induced vasodilation in the rat kidney. Hypertension. 1995; 25:790-5. [PubMed: 7721434]

27. Epigenomics Roadmap. et al. Integrative analysis of 111 reference human epigenomes. Nature. 2015; 518:317-30. [PubMed: 25693563]

28. Consortium EP. An integrated encyclopedia of DNA elements in the human genome. Nature. 2012; 489:57-74. [PubMed: 22955616]

29. Maurano MT, et al. Systematic localization of common disease-associated variation in regulatory DNA. Science. 2012; 337:1190-5. [PubMed: 22955828]

30. Welter D, et al. The NHGRI GWAS Catalog, a curated resource of SNP-trait associations. Nucleic Acids Res. 2014; 42:D1001-6. [PubMed: 24316577]

31. Trynka G, et al. Chromatin marks identify critical cell types for fine mapping complex trait variants. Nat Genet. 2013; 45:124-30. [PubMed: 23263488]

32. Ernst J, et al. Mapping and analysis of chromatin state dynamics in nine human cell types. Nature. 2011; 473:43-9. [PubMed: 21441907]

33. Segre AV, et al. Common inherited variation in mitochondrial genes is not enriched for associations with type 2 diabetes or related glycemic traits. PLoS Genet. 2010; 6

34. Pers TH, et al. Biological interpretation of genome-wide association studies using predicted gene functions. Nat Commun. 2015; 6:5890. [PubMed: 25597830]

35. Giresi PG, Kim J, McDaniell RM, Iyer VR, Lieb JD. FAIRE (Formaldehyde-Assisted Isolation of Regulatory Elements) isolates active regulatory elements from human chromatin. Genome Res. 2007; 17:877-85. [PubMed: 17179217]

36. Stergachis $\mathrm{AB}$, et al. Conservation of trans-acting circuitry during mammalian regulatory evolution. Nature. 2014; 515:365-70. [PubMed: 25409825]

37. Mancia G, et al. 2013 ESH/ESC guidelines for the management of arterial hypertension: the Task Force for the Management of Arterial Hypertension of the European Society of Hypertension (ESH) and of the European Society of Cardiology (ESC). Eur Heart J. 2013; 34:2159-219. [PubMed: 23771844]

38. Lifton, R.; Somlo, S.; Giebisch, G.; Seldin, D. Genetic Diseases of the Kidney. Academic Press; 2009.

39. Coffman TM, Crowley SD. Kidney in hypertension: guyton redux. Hypertension. 2008; 51:811-6. [PubMed: 18332286]

40. Kim $\mathrm{CH}$, et al. Mutations in the dopamine beta-hydroxylase gene are associated with human norepinephrine deficiency. American journal of medical genetics. 2002; 108:140-7. [PubMed: 11857564]

41. Deinum J, et al. DBH gene variants that cause low plasma dopamine beta hydroxylase with or without a severe orthostatic syndrome. Journal of medical genetics. 2004; 41:e38. [PubMed: 15060114]

42. Ghofrani HA, et al. Riociguat for the treatment of pulmonary arterial hypertension. N Engl J Med. 2013; 369:330-40. [PubMed: 23883378]

44. Yang J, Lee SH, Goddard ME, Visscher PM. GCTA: a tool for genome-wide complex trait analysis. Am J Hum Genet. 2011; 88:76-82. [PubMed: 21167468]

45. Yang J, et al. Conditional and joint multiple-SNP analysis of GWAS summary statistics identifies additional variants influencing complex traits. Nat Genet. 2012; 44:369-75. S1-3. [PubMed: 22426310]

46. Penninx BW, et al. The Netherlands Study of Depression and Anxiety (NESDA): rationale, objectives and methods. Int J Methods Psychiatr Res. 2008; 17:121-40. [PubMed: 18763692]

47. Boomsma DI, et al. Netherlands Twin Register: from twins to twin families. Twin Res Hum Genet. 2006; 9:849-57. [PubMed: 17254420]

48. Visscher PM, Benyamin B, White I. The use of linear mixed models to estimate variance components from data on twin pairs by maximum likelihood. Twin Res. 2004; 7:670-4. [PubMed: 15607018] 
49. Romanoski CE, et al. Network for activation of human endothelial cells by oxidized phospholipids: a critical role of heme oxygenase 1. Circ Res. 2011; 109:e27-41. [PubMed: 21737788]

50. Koopmann TT, et al. Genome-wide identification of expression quantitative trait loci (eQTLs) in human heart. PLoS One. 2014; 9:e97380. [PubMed: 24846176]

51. Fairfax BP, et al. Innate immune activity conditions the effect of regulatory variants upon monocyte gene expression. Science. 2014; 343:1246949. [PubMed: 24604202]

52. Ramasamy A, et al. Genetic variability in the regulation of gene expression in ten regions of the human brain. Nat Neurosci. 2014; 17:1418-28. [PubMed: 25174004]

53. Maurano MT, et al. Systematic localization of common disease-associated variation in regulatory DNA. Science. 2012; 337:1190-5. [PubMed: 22955828]

54. Welter D, et al. The NHGRI GWAS Catalog, a curated resource of SNP-trait associations. Nucleic Acids Res. 2014; 42:D1001-6. [PubMed: 24316577]

55. Trynka G, et al. Chromatin marks identify critical cell types for fine mapping complex trait variants. Nat Genet. 2013; 45:124-30. [PubMed: 23263488]

56. Segre AV, et al. Common inherited variation in mitochondrial genes is not enriched for associations with type 2 diabetes or related glycemic traits. PLoS Genet. 2010; 6

57. Pers TH, et al. Biological interpretation of genome-wide association studies using predicted gene functions. Nat Commun. 2015; 6:5890. [PubMed: 25597830]

58. Magi R, Morris AP. GWAMA: software for genome-wide association meta-analysis. BMC Bioinformatics. 2010; 11:288. [PubMed: 20509871]

59. Johnson T. Efficient Calculation for Multi-SNP Genetic Risk Scores. ASHG 2012 Annual Meeting. poster presentation. 


\section{SUMMARY STATISTICS}

Full summary statistics ( $P$ values) are in the online version of the paper (file “ICBPCMfinalMeta.csv.zip”). 


\section{SBP}
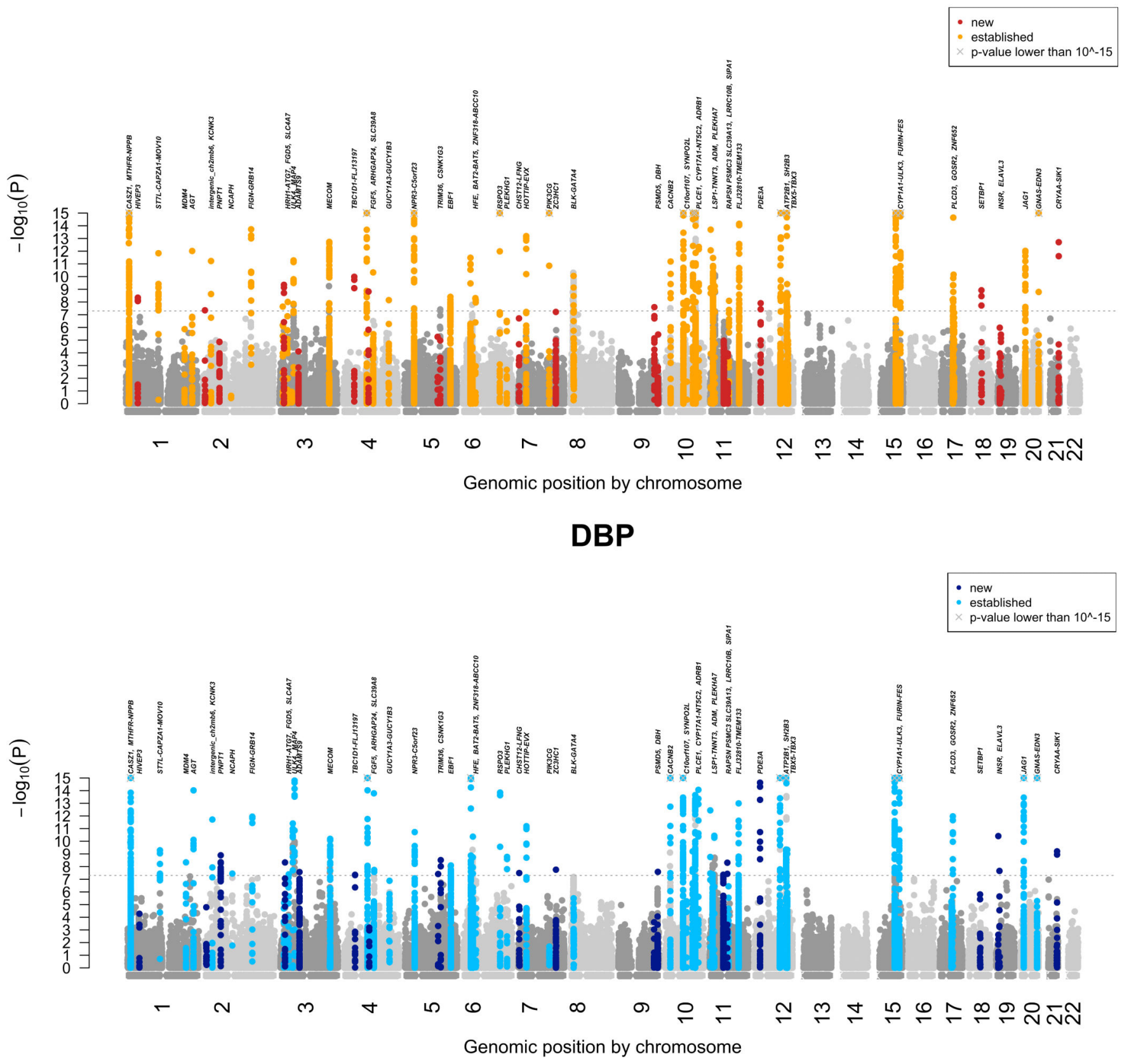

Figure 1. Manhattan plots for SBP and DBP from the stage 4 Cardio-MetaboChip-wide metaanalysis

$P$ values (expressed as $-\log _{10} P$ ) are plotted by physical genomic position labeled by chromosome. SNPs in new loci (3.5MB window around the index SNP), identified in this study, are labeled in dark red (SBP) or dark blue (DBP); SNPs in previously known loci are labeled in orange (SBP) or light blue (DBP). The locus names are indicated. The grey crosses indicate genomic positions at which the y-axis was truncated (SNPs with $P<10^{-15}$ ). 
A) Narrow DHS region definition

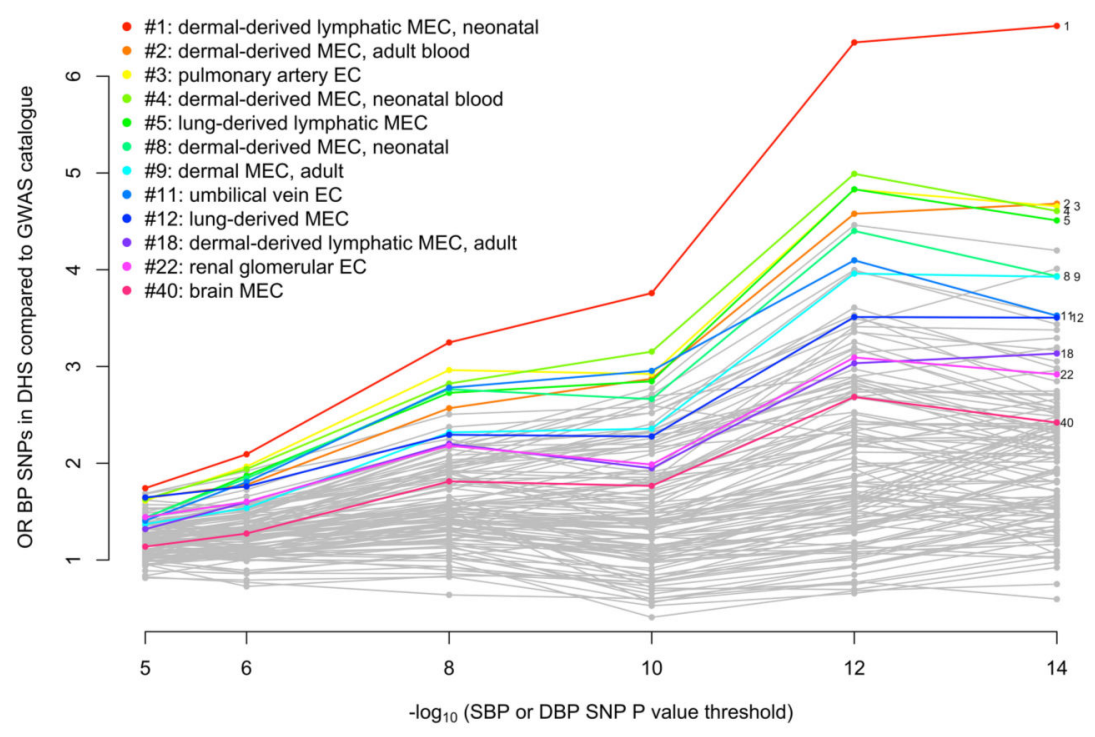

B) Broad DHS region definition

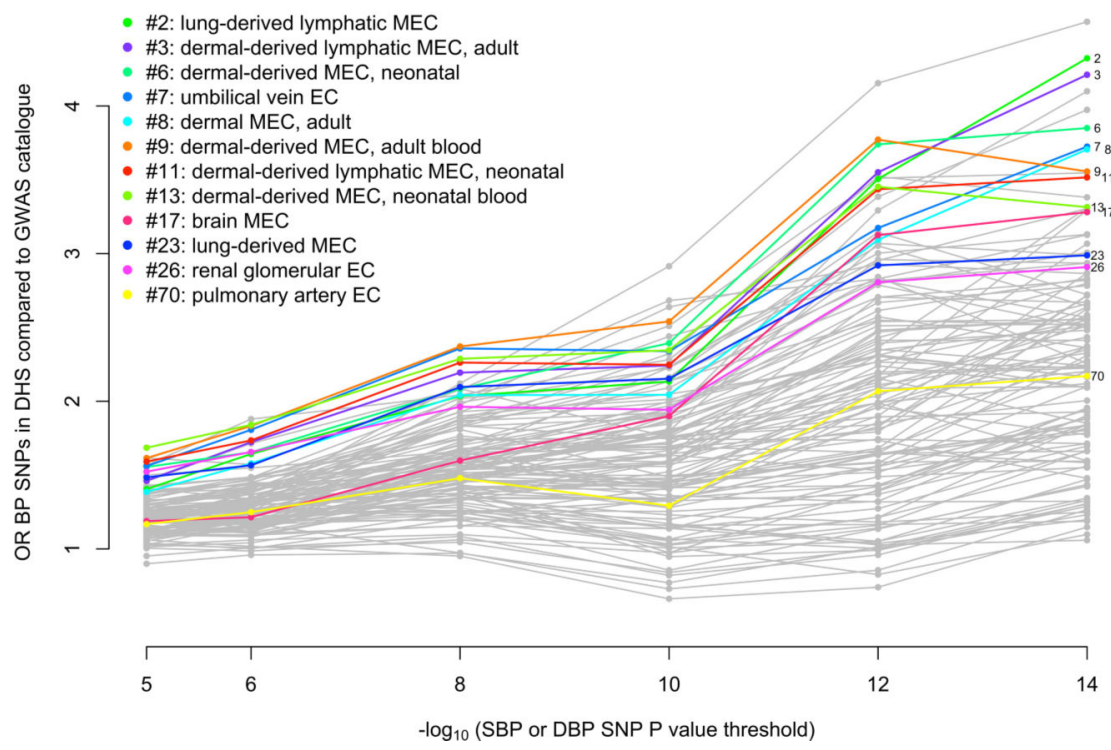

Figure 2. Enrichment of DNAse hypersensitive sites among BP loci in different cell-types Enrichment analyses of SBP or DBP associated loci according to discovery $P$ value using narrow peaks (panel A) or broad peaks (panel B). SNPs were selected according to different $P$ value cutoffs (x-axis) and a fold enrichment of overlap with DNAse hypersensitive sites compared to unrelated GWAS SNPs was calculated (y-axis) (see Supplementary Note). The 12 endothelial cell-lines are indicated in color and for each endothelial cell-type the rank using the $10^{-14} P$ value cutoff is indicated. EC denotes endothelial cells. 


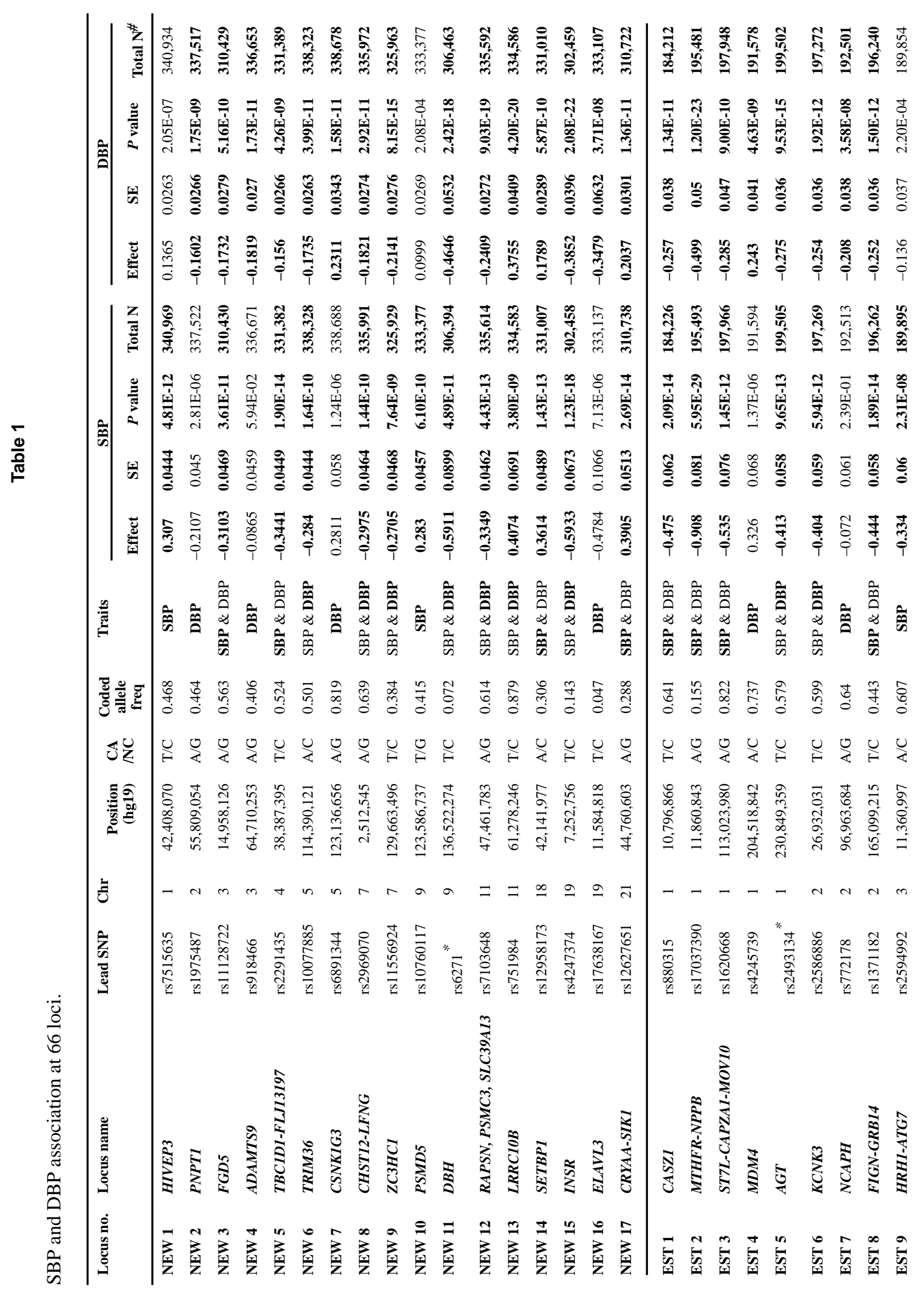




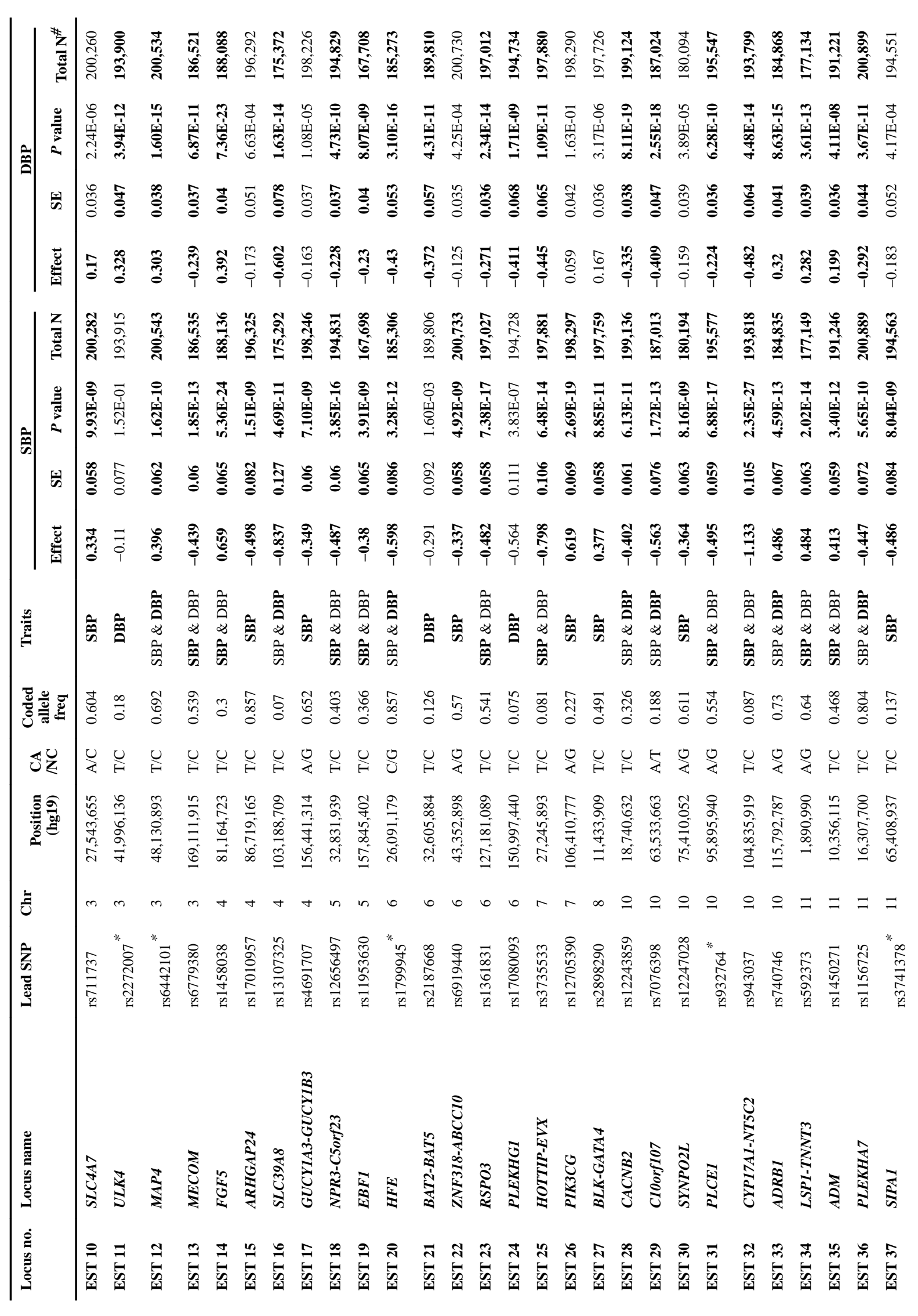




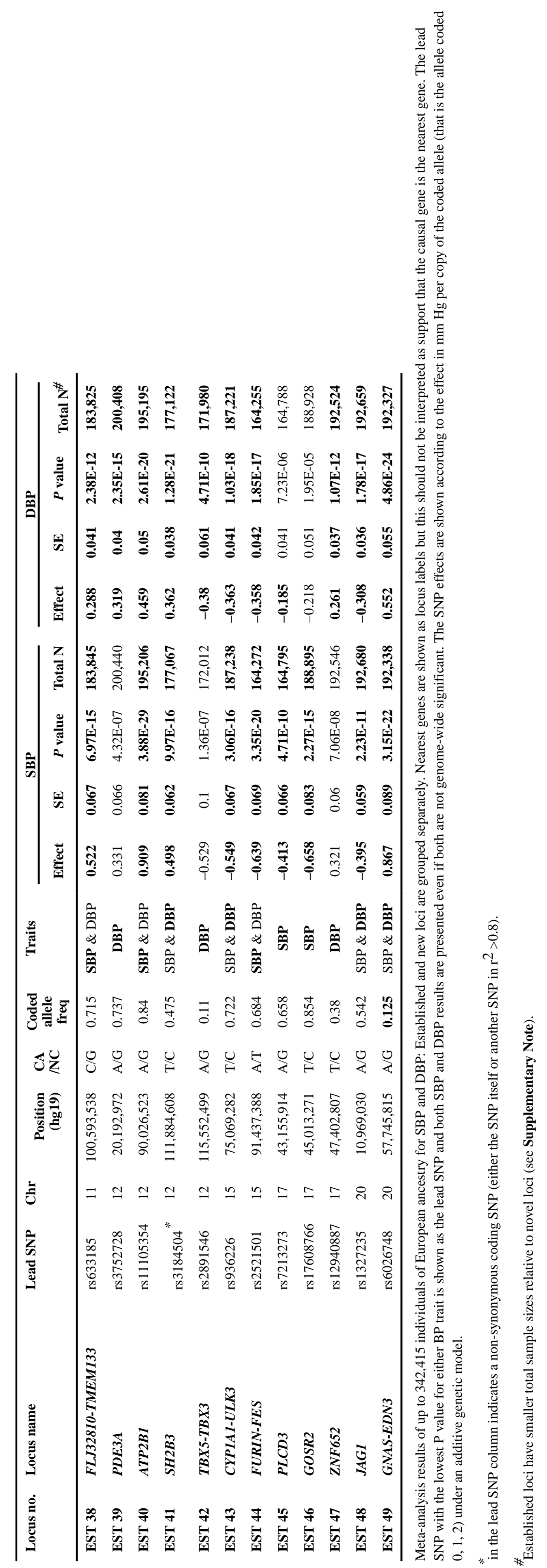




\section{Table 2}

Overview of novel and known BP variant properties.

\begin{tabular}{llll}
\hline & 17 new loci & 49 established loci & 66 loci \\
\hline Minor allele frequency (mean, range) & $32.1 \%[5 \%-50 \%]$ & $28.9 \%[7 \%-49 \%]$ & $29.8 \%[5 \%-50 \%]$ \\
Effect size SBP [mmHg] (range, mean) & $0.09-0.59,0.34$ & $0.07-1.13,0.5$ & $0.07-1.13,0.46$ \\
Effect size DBP [mmHg] (range, mean) & $0.1-0.46,0.23$ & $0.06-0.60,0.3$ & $0.06-0.6,0.28$ \\
Variance explained SBP & $0.52 \%$ & $2.95 \%$ & $3.46 \%$ \\
Variance explained DBP & $0.58 \%$ & $2.78 \%$ & $3.36 \%$ \\
\hline
\end{tabular}

Key characteristics of the novel and established BP loci are shown. MAF and effect size estimates are derived from the Cardio-MetaboChip data. Variance explained estimates are estimated from one large study (Supplementary Note). Novel loci are classified as previously unknown to be linked to BP by a systematic PubMed review of all genes in a 200kb window (Supplementary Note). 


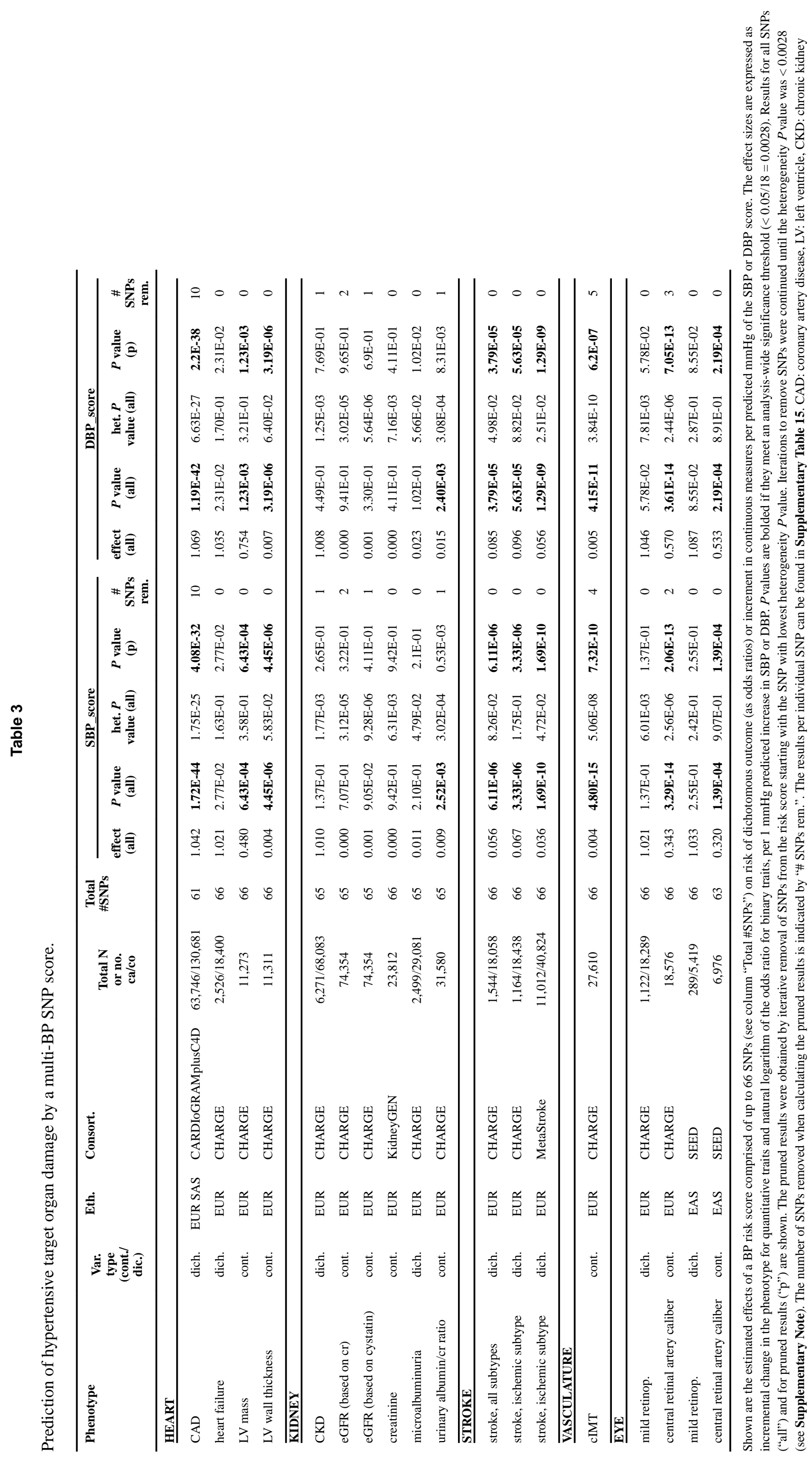


